\title{
Equilibrium and out of equilibrium phase transitions in systems with long range interactions and in $2 \mathrm{D}$ flows
}

\author{
Freddy Bouchet*, Julien Barré ${ }^{\dagger}$ and Antoine Venaille** \\ *Institut Non Lineaire de Nice, INLN, UMR 6618, CNRS, UNSA, 1361 route des lucioles, 06560 \\ Valbonne - Sophia Antipolis, France \\ $\dagger$ Université de Nice-Sophia Antipolis, Laboratoire J. A. Dieudonné, Parc Valrose, 06108 Nice \\ Cedex 02, France \\ ** Coriolis-LEGI, 21 avenue des Martyrs, 38000 Grenoble, France
}

\begin{abstract}
In self-gravitating stars, two dimensional or geophysical flows and in plasmas, long range interactions imply a lack of additivity for the energy; as a consequence, the usual thermodynamic limit is not appropriate. However, by contrast with many claims, the equilibrium statistical mechanics of such systems is a well understood subject. In this proceeding, we explain briefly the classical approach to equilibrium and non equilibrium statistical mechanics for these systems, starting from first principles. We emphasize recent and new results, mainly a classification of equilibrium phase transitions, new unobserved equilibrium phase transition, and out of equilibrium phase transitions. We briefly discuss what we consider as challenges in this field.
\end{abstract}

Keywords: Long range interactions, Inequivalence of ensembles, Kinetic theory, Out of equilibrium phase transitions, Two dimensional turbulence, Geophysical flows.

PACS: 05.20.Dd, 05.20.Gg, 05.70.Fh, 05.70.Ln

\section{INTRODUCTION}

In a large number of physical systems, any single particle feels a potential dominated by interactions with far away particles: this is our definition of long range interactions. In a system with algebraic decay of the inter-particle potential $V(r) \underset{r \rightarrow \infty}{\sim} r^{\alpha}$, this occurs when $\alpha$ is less than the dimension of the system (these interactions are sometimes called "non-integrable"). Then the energy is not additive, as the interaction of any subpart of the system with the whole is not negligible with respect to the internal energy of this given part.

Self gravitating stars, after the discovery of negative specific heat in [1], have played a very important historical role, by emphasizing the peculiarities in the statistical mechanics of systems with long range interactions. Besides astrophysical self gravitating systems [2, 3, 4, 5, 6, 7, 8, 9, 10, 11], the main physical examples of non-additive systems with long range interactions are two-dimensional or geophysical fluid dynamics [12, 13, 14, 15, 16, 17] and a large class of plasma effective models [18, 19, 20, 21]. Spin systems [22] and toy models with long range interactions [23, 24, 25] have also been widely studied. The links between these different subjects have been emphasized recently [23]. 
In these systems, the most prominent and interesting physical phenomenon is the self organization of the particles, or of the velocity field. This leads to coherent clouds of particles in plasma physics, to galaxies and globular clusters in astrophysics and to large scale jets and vortices in two dimensional or geophysical flows. Given the large number of particles or of degrees of freedom, it is tempting to adopt a statistical approach in order to describe these phenomena. The statistical description of such a self organization, both at the levels of equilibrium situations and relaxation towards equilibrium (kinetic theories), is a classical, long studied field. One of the aim of this proceeding is to insist on the vitality of this old subject and to stress new advances and remaining issues. By contrast, the out of equilibrium statistical mechanics of such phenomena is still in its infancy, and few studies have been devoted to it. We emphasize the importance of such studies for real applications, as most plasma and geophysical physical phenomena are out of equilibrium. We also describe some recent very suggestive results.

Both equilibrium and out of equilibrium phase transitions play a key role in our understanding of physics, because they separate regions of parameter space with qualitatively different behaviors. Very naturally, a large part of our studies will be devoted to phase transitions. We will especially stress the peculiar association of phase transitions with negative specific heat and statistical ensemble inequivalence in systems with long range interactions. We also insist on recently observed out of equilibrium phase transtions, in the context of two dimensional flows. Finally, we describe our personal guesses for what may be the challenges and interesting issues in the field of systems with long range interactions. We hope this could open new discussions, although we are conscious that such guesses are necessarily biased by personal prejudices. We actually sincerely hope that future researches will be much richer than what we describe. The article is organized in three main sections: equilibrium, relaxation to equilibrium and kinetic theories, non equilibrium stationary states.

Equilibrium. Long range interacting systems are known to display peculiar thermodynamic behaviors. As additivity is often seen as a cornerstone of usual statistical mechanics and thermodynamics, it is sometimes written in textbooks or articles that "statistical mechanics or thermodynamics do not apply to systems with long range interactions". In this paper, we argue on the contrary that usual tools and ideas of statistical mechanics do apply to such systems, both at equilibrium and out of equilibrium. However, reviewing a variety of recent works, we will show that a careful application of these tools reveals truly unusual and fascinating behaviors, absent from the world of short range interacting systems.

After a brief introduction on the unusual negative specific heat and other peculiar thermodynamical phenomena, we discuss the usual assumptions of equilibrium statistical mechanics and their interpretation in systems with long range interactions. Based on the assumption of equal probability of any configuration with a given energy, we then explain why the Boltzmann-Gibbs entropy actually measures the probability to observe a given distribution function. This relies on our ability to prove large deviations results for such systems. The result of this analysis is that microcanonical and canonical ensembles of systems with long range interactions are described by two dual variational problems. We explain why such variational problems lead to possible generic ensemble inequivalence, and to a richer zoology of phase transitions than in usual systems. A 
natural question then arises: do we know all possible behaviors stemming from long range interactions, and, if not, what are the possible phenomenologies? We answer this question by discussing a classification of all microcanonical and canonical phase transitions, in long range interacting systems, with emphasis on situations of ensemble inequivalence [26]. Very interestingly many possible phase transitions and situations of ensemble inequivalence have not been observed yet. We then describe, for two dimensional flows, the first observation of appearance of ensemble inequivalence associated to bicritical and azeotropy phase transitions.

Kinetic theories and relaxation toward equilibrium. Because systems with long range interactions relax very slowly towards equilibrium, or because they can be forced by external field, the study of out of equilibrium situations is physically essential. During the past century, there have been many attempts to find a general formalism for out of equilibrium statistical mechanics, which would give the equivalent of the Gibbs picture for out of equilibrium states. Unfortunately, as recognized by most of the statistical mechanics community, until now any such attempt failed. This is mainly due to the fact that our knowledge of out of equilibrium situations can not be parameterized by a small number of macroscopic quantities, playing the same role as dynamical invariants for the equilibrium theory. Then out of equilibrium statistical mechanics must be addressed by a case by case careful examination of dynamics, using some appropriate probabilistic description.

For relaxation to equilibrium of Hamiltonian systems with long range interactions, standard tools have been developed, mainly kinetic theory. In the introductory paragraph, we briefly explain the basic ideas of kinetic theories. In the following, we first stress the role of a Vlasov description for small time, and then the role of Lenard-Balescu equation (also called collisional Boltzmann equation in the context of self-gravitating systems) for larger time. We also briefly review the recent application of Lynden-Bell equilibrium statistical mechanics for the Vlasov equation to simple one dimensional models. We also discuss new recent results for the kinetic theory of such systems. The first is the generic existence, for the one particle stochastic process, of anomalous diffusion and of long relaxation times. We guess that the implications of such a result for the validity of the kinetic approach has not been well appreciated up to now. The second class of results deals with the time of validity for the Vlasov approximation and with the typical time needed to observe relaxation towards equilibrium. One of the most striking result is that the Lenard Balescu operator vanishes identically for one dimensional systems. This explains the existence of anomalous scaling laws for the relaxation towards equilibrium in models like the HMF model.

Non equilibrium stationary states (NESS). Another class of out of equilibrium problems is the study of systems with long range interactions subjected to small nonHamiltonian forces and to weak dissipation. Such a framework is actually the most relevant one for many physical applications. We will emphasize its interest for geophysical flows, like for instance simplified models of ocean currents. 


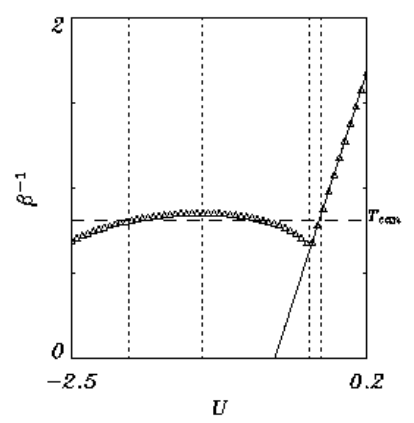

FIGURE 1. Caloric curve (temperature $T=\beta^{-1}$ as a function of the energy $U$ ) for the SGR model, for $\varepsilon=10^{-2}$. The energies corresponding to the dashed vertical lines are referred to, from left to right, as $U_{\text {low }}, U_{\text {top }}, U_{c}$, and $U_{\text {high }}$. The decreasing temperature between $U_{\text {top }}$ and $U_{c}$ characterizes a range of negative specific heat and thus of ensemble inequivalence. At $U_{c}$, there is a microcanonical second order phase transition associated with the canonical first order phase transition (please see the text for a detailed explanation). Such a behavior is linked to the existence of tricritical points in both statistical ensembles (see figure 2). A classification of all possible routes to ensemble inequivalence is briefly described in section 2.4 or in [26].

The average balance between forcing and dissipation usually leads to statistically stationary states, the properties of which may be studied experimentally, numerically and theoretically. As there is no detailed balance, the system is maintained out of equilibrium. The fluxes of the Hamiltonian conserved quantities then become essential physical variables.

We show in this last section, that this leads, in the context of two dimensional flows, to very interesting out of equilibrium phase transitions. We believe that the study of the statistical mechanics of such non equilibrium stationary states and phase transitions, in other systems with long range interactions, is one of the main challenges in this field.

\section{EQUILIBRIUM STATISTICAL MECHANICS OF SYSTEMS WITH LONG RANGE INTERACTIONS}

\subsection{Peculiarities of thermodynamics of systems with long range interactions}

For systems with long range interactions, the most intriguing thermodynamical property is the generic occurrence of statistical ensemble inequivalence and negative specific heat. Such possibilities have first been recognized and studied in the context of self gravitating systems [1, 27, 11]. Afterwards, ensemble inequivalence and negative specific heat have been observed or predicted in a number of different physical systems: two dimensional turbulence [12, 28, 29], plasma physics [28, 19], spin systems or toy models [22, 25], or self gravitating systems in situations different from the simple initial case $[7,5,3,4,6,30,9]$. A detailed description of each of these cases is provided in [26]. 

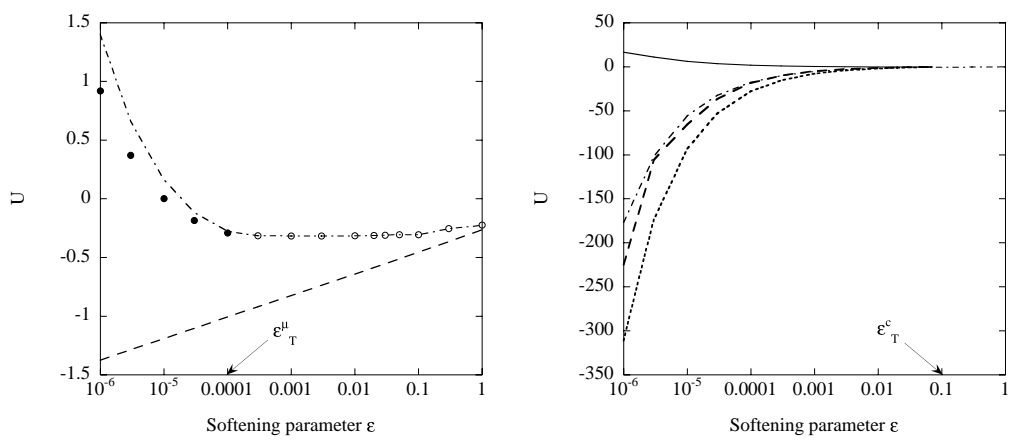

FIGURE 2. Phase diagrams for the SGR model. Left: for each value of $\varepsilon$, the critical energies where a microcanonical phase transition occurs are represented by black points or circles. When the softening parameter is varied, at the tricritical point $\varepsilon=\varepsilon_{T}^{\mu}$, first order phase transitions (black points) change to second order phase transitions (circles). Right: the bottom dashed line and black line represent respectively $U_{\text {low }}$ (see fig. 1) and $U_{\text {high }}$. The difference $U_{\text {high }}-U_{\text {low }}$ is the energy jump associated to a first order phase transition in the canonical ensemble. This jump decreases to zero at canonical tricritical point $\varepsilon_{T}^{c}$ where the canonical first order phase transition changes to a canonical second order phase transition. The tricritical points have different energies and $\varepsilon$ values in both ensembles.

To motivate the following development, let us give an example where ensemble inequivalence appears in an unusual way. We discuss the equilibrium properties of the Self Gravitating Ring (SGR) model, a toy model for self gravitating systems. Whereas we mainly present here its equilibrium properties, we stress that this system is very interesting also from a dynamical point of view, as it shows a number of out of equilibrium quasi-stationary states [31, 32].

The Hamiltonian of the SGR model is: $H=\frac{1}{2} \sum_{i=1}^{N} p_{i}^{2}-\frac{1}{2} \sum_{i, j=1}^{N} \frac{1}{\sqrt{2}} \frac{1}{\sqrt{1-\cos \left(\theta_{i}-\theta_{j}\right)+\varepsilon}}$. Particles are constrained on a ring $\left(0 \leq \theta_{i} \leq 2 \pi\right)$. The angles $\theta_{i}$ are conjugate to the momenta $p_{i} . \varepsilon$ is a small scale softening of the gravitational interaction. We study the phase transitions of this system and how they evolve when $\varepsilon$ is varied. Please see [30] for a detailed discussion.

Figure 11 shows the caloric curve $T(U)$ for $\varepsilon=10^{-2}$, where $T$ is the temperature and $U$ is the energy. For $U_{\text {top }}<U<U_{c}$ the temperature is decreasing. The specific heat $C=d U / d T$ is thus negative in this area, showing that statistical ensembles are not equivalent (in the canonical ensemble, the specific heat is always positive). The horizontal dashed line is the Maxwell construction, which links microcanonical and canonical ensembles. From it one sees that in the canonical ensemble, when $\beta$ is varied, there is a first order phase transition characterized by an energy jump between the values $U_{\text {low }}$ and $U_{\text {high }}$. This is a common feature in case of ensemble inequivalence.

What is less common is the concomitant existence of a second order phase transition in the microcanonical ensemble, at the energy $U_{c}$. At this point, the temperature $T$ is continuous, whereas its derivative is discontinuous as it is clear from the curve. In figure 2, we show that this type of ensemble inequivalence, with the coexistence of a first order canonical first order phase transition and of a microcanonical second order phase transition, is linked to the existence of a tricritical point in both ensembles. 
The SGR model displays one possible route to ensemble inequivalence, out of several others. However, there are some constraints on the possible phenomenologies. For instance, at a second order phase transition, the negative specific heat jump must be positive. By contrast, the temperature jumps at a discontinuity associated with a first order microcanonical phase transition must be negative (this means that when energy is increased the system has a negative temperature jump). Summarizing all these constraints yields a classification [26] of all possible ensemble inequivalences and their links with phase transitions. To prepare the discussion of this classification, we recall now the main hypothesis and definitions of statistical equilibrium, in the context of systems with long range interactions.

\subsubsection{Additivity extensivity and thermodynamic limit}

When studying statistical mechanics of non additive systems, the first problem one has to deal with is the inadequacy of the thermodynamic limit $(N \rightarrow \infty, V \rightarrow \infty-V$ is the volume-, with $N / V$ kept constant). Indeed, what is physically important in order to understand the behavior of large systems, is not really to study the large $N$ limit, but rather to obtain properties that do not depend much on $N$ for large $N$ (the equivalent of intensive variables). For short range interacting systems, this is achieved through the thermodynamic limit; for non additive systems, the scaling limit to be considered is different and depends on the problem. Let us consider $N$ particles which dynamics is described by the Hamiltonian

$$
H_{N}=\frac{1}{2} \sum_{i=1}^{N} p_{i}^{2}+\frac{c}{2} \sum_{i, j=1}^{N} V\left(x_{i}-x_{j}\right)
$$

where $c$ is a coupling constant. The thermodynamic limit in this case amounts to send $N$ and the volume to infinity, keeping density and $c$ constant. If $V(x)$ decreases fast enough so that interactions for a particle come mainly from the first neighbors, then increasing $N$ at constant density has almost no effect on the bulk, and physical properties are almost independent of $N$ : the thermodynamic limit is appropriate. This is wrong of course if the potential for a particle is dominated by the influence of far away particles. The appropriate scaling in this case may be as follows: fixed volume, $c \propto 1 / N^{2}$, and $N \rightarrow \infty$ (others equivalent combinations are possible, as the one given below for self gravitating particles).

The best known example of such a special scaling concerns self gravitating stars, for which the ration $M / R$ is usually kept constant, where $M$ is the total mass and $R$ is the system's radius (thermodynamic limit would be $M / R^{3}$ constant). Another toy example is given and studied for instance in [33]. This type of scaling is also the relevant one for point vortices in two dimensional and geophysical turbulence, where the total volume and total vorticity have to be kept fixed, but divided in smaller and smaller units. Let us note for completeness that in some cases, the thermodynamic limit is appropriate in presence of long range interactions, for instance when some screening is involved [34]; we shall exclude these cases in the following. 
According to the above discussion, let us rewrite Eq. 1 using the convenient scaling $c= \pm 1 / N$ :

$$
H_{N}=\frac{1}{2} \sum_{i=1}^{N} p_{i}^{2} \pm \frac{1}{2 N} \sum_{i, j=1}^{N} V\left(x_{i}-x_{j}\right)
$$

This classical scaling of the coupling parameter is called the Kac's prescription ([35]) or sometimes the mean field scaling (see for instance [36]). Within this scaling, taking the limit $N \rightarrow \infty$ with all other parameters fixed (fixed volume for instance), the sum over $i$ and $j$ is clearly of order $N^{2}$, and the energy per particle $H_{N} / N$ is intensive. This scaling is also the relevant one in order to obtain the collisionless Boltzmann equation, for the dynamics, in the large $N$ limit. We will use Eq. 2 in the following.

\subsubsection{The microcanonical and canonical ensembles}

We suppose that the energy $E$ of our system is known, and consider the microcanonical ensemble. In this statistical ensemble all phase space configurations with energy $E$ have the same probability; the associated microcanonical measure is then

$$
\mu_{N}=\frac{1}{\Omega_{N}(E)} \prod_{i=1}^{N} d x_{i} d p_{i} \delta\left(H_{N}\left(\left\{x_{i}, p_{i}\right\}\right)-E\right),
$$

where $\Omega_{N}(E)$ is the volume of the energy shell in the phase space $\Omega_{N}(E) \equiv$ $\int \prod d x_{i} d p_{i} \delta\left(H_{N}\left(\left\{x_{i}, p_{i}\right\}\right)-E\right)$. We consider here the energy as the only parameter, however generalization of the following discussion to other quantities conserved by the dynamics is straightforward.

The only hypothesis of equilibrium statistical mechanics is that averages with respect to $\mu_{N}$ will correctly describe the macroscopic behavior of our system. This hypothesis is usually verified after a sufficiently long time, when the systems has "relaxed" to equilibrium.

The Boltzmann entropy per particle is defined as

$$
S_{N}(E) \equiv \frac{1}{N} \log \Omega_{N}(E)
$$

In the following, we will justify that in the long range thermodynamic limit, the entropy per particle $S_{N}(E)$ has a limit:

$$
S_{N}(E) \underset{N \rightarrow \infty}{\rightarrow} S(E)
$$

The canonical ensemble is defined similarly, using the canonical measure

$$
\mu_{c, N}=\frac{1}{Z_{N}(E)} \prod_{i=1}^{N} d x_{i} d p_{i} \exp \left[-\beta H_{N}\left(\left\{x_{i}, p_{i}\right\}\right)\right],
$$

with the associated partition function $Z_{N}(\beta) \equiv \int \prod d r_{i} d p_{i} \exp \left[-\beta H_{N}\left(\left\{x_{i}, p_{i}\right\}\right)\right]$ and free energies $F_{N}(\beta) \equiv \frac{1}{N} \log Z_{N}(\beta)$ and $F_{N}(\beta) \underset{N \rightarrow \infty}{\rightarrow} F(\beta)$ 


\subsection{Large deviation results}

\subsubsection{Justification of the Boltzmann-Gibbs entropy}

Let us consider the particle distribution on $\mu$-space $f(x, p)(f(x, p) d x d p$ is the probability to observe a particle with position $x$ and momentum $p) . f$ defines a macrostate as many microscopic states correspond to a given $f$. As explained in the previous paragraph, the hypothesis of usual statistical mechanics is that all microscopic states with a given energy $E$ are equiprobable. Given this uniformity in phase space, we address the question: what is the number of microscopic states having the distribution $f$ ?

It is a classical combinatorial result to show that the logarithm of number of microscopic states corresponding to a distribution $f$ is given by

$$
s[f]=-\int d x d p f \log f
$$

where $s$ is sometimes called the Boltzmann-Gibbs entropy. It is the Boltzmann entropy associated to the macrostate $f$, in the sense that it counts the number of microstates corresponding to $f$. We stress that no other functional has this probabilistic meaning, and that this property is independent of the Hamiltonian.

Thanks to the long range nature of the interaction, for most configurations, the energy per particle can be expressed in term of the distribution function $f$, using

$$
h(f) \underset{N \rightarrow \infty}{\sim} \int \frac{p^{2}}{2} f(x, p) d x d p+\int d x_{1} d p_{1} d x_{2} d p_{2} f\left(x_{1, p_{1}}\right) f\left(x_{2}, p_{2}\right) V\left(x_{1}-x_{2}\right) .
$$

This mean field approximation for the energy allows to conclude that the equilibrium entropy is given by

$$
S_{N}(E)=\log \left(\Omega_{N}(E)\right) \underset{N \rightarrow \infty}{\sim} N S(E) \text { with } S(E)=\sup _{f}\{s(f) \mid h(f)=E\}
$$

In the limit of a large number of particles, the mean field approximation Eq. (B) and its consequence the variational problem (\#) have been justified rigorously for many systems with long range interactions. The first result assumes a smooth potential $V$ and has been proved by [36], see also the works by Hertel and Thirring on the self gravitating fermions [27].

\subsubsection{Large deviations}

We explained why the Boltzmann-Gibbs entropy is the correct one to describe the probability of a given $f$. Large deviations provide a useful tool to obtain similar results in a wider context. We refer to the very interesting contributions of Ellis and coworkers ([37, 29, 38, 39, 40]). We also refer to [41] for a simple detailed explanation of many large deviations results in the context of long range interacting systems.

In a first step one describes the system at hand by a macroscopic variable; this may be a coarse-grained density profile $f$, a density of charges in plasma physics, a 
magnetization profile for a magnetic model. In the following, we will generically call this macroscopic variable $m$; it may be a scalar, a finite or infinite dimensional variable.

One then associates a probability to each macrostate $m$. Large deviation theory comes into play to estimate $\Omega(m)$, the number of microstates corresponding to the macrostate $m$ :

$$
\log \left(\Omega_{N}(m)\right) \underset{N \rightarrow \infty}{\sim} N s(m) .
$$

This defines the entropy $s(m)$.

In a second step, one has to express the constraints (energy or other dynamical invariants) as functions of the macroscopic variable $m$. In general, it is not possible to express exactly $H$; however, for long range interacting systems, one can define a suitable approximating mean field functional $h(m)$, as in Eq. (3).

Having now at hand the entropy and energy functionals, one can compute the microcanonical density of states $\Omega(E)$ ([37]): the microcanonical solution is simply given by the variational problem

$$
\log \left(\Omega_{N}(E)\right) \underset{N \rightarrow \infty}{\sim} N S(E) \text { with } S(E)=\sup _{m}\{s(m) \mid h(m)=E\}
$$

In the canonical ensemble, similar considerations lead to the conclusion that the free energy and the canonical equilibrium are given by the variational problem

$$
\log \left(Z_{N}(\beta)\right) \underset{N \rightarrow \infty}{\sim} N F(\beta) \text { with } F(\beta)=\inf _{m}\{-s(m)+\beta h(m)\}
$$

We insist that this reduction of the microcanonical and canonical calculations to the variational problems (5) and (6) is in many cases rigorously justified.

\subsection{Ensemble equivalence and simplification of variational problems}

As discussed in the previous section, the microcanonical and canonical equilibrium states are, most of the times, given by (5) and (6) respectively. These two variational problems are dual ones: the canonical one is obtained from the microcanonical one by relaxing a constraint. In the following section, we discuss the mathematical links between two such dual variational problems. We then apply this to characterize ensemble equivalence, and we use it to prove relations between classes of variational problems.

\subsubsection{Relations between constrained and relaxed variational problems}

It is possible to state some general results about the variational problems (5) and (6), independently of the precise form of the functions $s$ and $h$ :

1. a minimizer $m_{c}$ of (6) is a minimizer of (5), with constraint $E=h\left(m_{c}\right)$.

2. a minimizer $m_{\mu}$ of (5) is a critical point of (6) for some $\beta$, but it is not always a minimizer: it is a minimizer of (6) if and only if $S$ coincides with its concave hull at point $E=h\left(m_{\mu}\right)$. Otherwise, it may be a local minimum, or a saddle point of (6). 
Such results are extremely classical. More detailed results in this context may be found in [37]. We also refer to [42] for a concise discussion and proof. The previous points immediately translate into the language of statistical mechanics, and provide a full characterization of ensemble inequivalence:

- A canonical equilibrium is always a microcanonical equilibrium for some energy $E$.

- A microcanonical equilibrium at energy $E$ is a canonical equilibrium for some temperature $1 / \beta$ if and only if $S$ coincides with its concave hull at energy $E$. Whenever $S$ coincides with its concave hull, we will say that the ensembles are equivalent; otherwise we will say they are not equivalent.

\subsubsection{Simpler variational problem for statistical equilibria}

In the previous paragraph, we have explained relations between solutions of a constrained variational problem and of the associated relaxed one. Using similar results and further theoretical considerations, it is possible to obtain much simpler variational problems than the natural microcanonical ones, for the equilibria of Euler and Vlasov equations [42]. We think that these new results provide essential simplifications that will be useful in many studies, we thus describe them in this section. However, from a physical point of view, these mathematical results may be viewed as technical, and we advise the non expert reader to skip this section at first reading.

When studying statistical equilibria of systems with long range interactions, one has to deal with variational problems with one or several constraints. In the case of the statistical mechanics of the Euler (resp. the Vlasov equation), there is actually an infinite number of Casimir's functional conservation laws, encoded in the initial distribution $d$ of the vorticity field (resp. the particle distribution function). This is a huge practical limitation. When faced with real phenomena, physicists can then either give physical arguments for a given type of distribution $d$ (modeler approach) or ask whether there exists some distribution $d$ with equilibria close to the observed field (inverse problem approach). However, in any case the complexity remains: the class of equilibria is huge.

In the following of this paragraph, we describe recent mathematical results which allow to relate the microcanonical equilibria to much simpler variational problems. From a physical point of view, this simplification is extremely interesting. We describe these results in the context of the equilibrium theory for the Euler equation (Robert Sommeria Miller theory [15, 14] or RSM theory), but the following results may be easily generalized to other cases like the statistical mechanics of the Vlasov equation. We refer to [42] for a more detailed discussion.

$>$ From a mathematical point of view, one has to solve a microcanonical variational problem (MVP): maximizing a mixing entropy $\mathscr{S}[\rho]=-\int_{\mathscr{D}} d^{2} x \int d \sigma \rho \log \rho$, with constraints on energy $E$ and vorticity distribution $d$

$$
S\left(E_{0}, d\right)=\sup _{\{\rho \mid N[\rho]=1\}}\left\{\mathscr{S}[\rho] \mid E[\bar{\omega}]=E_{0}, D[\rho]=d\right\}(\mathrm{MVP}) .
$$


$\rho(\mathbf{x}, \sigma)$ depends on space $\mathbf{x}$ and vorticity $\sigma$ variables.

During recent years, authors have proposed alternative approaches, which led to practical and/or mathematical simplifications in the study of such equilibria. As a first example, Ellis, Haven and Turkington [29] proposed to treat the vorticity distribution canonically (in a canonical statistical ensemble). From a physical point of view, a canonical ensemble for the vorticity distribution would mean that the system is in equilibrium with a bath providing a prior distribution of vorticity. As such a bath does not exist, the physically relevant ensemble remains the one based on the dynamics: the microcanonical one. However, the Ellis-Haven-Turkington approach is extremely interesting as it provides a drastic mathematical and practical simplification to the problem of computing equilibrium states. A second example, largely popularized by Chavanis [43, 44], is the maximization of generalized entropies. Both the prior distribution approach of Ellis, Haven and Turkington or its generalized thermodynamics interpretation by Chavanis lead to a second variational problem: the maximization of Casimir's functionals, with energy constraint (CVP)

$$
C\left(E_{0}, s\right)=\inf _{\omega}\left\{\mathscr{C}_{s}[\omega]=\int_{\mathscr{D}} s(\omega) d^{2} x \mid E[\omega]=E_{0}\right\}(\mathrm{CVP})
$$

where $\mathscr{C}_{s}$ are Casimir's functionals, and $s$ a convex function (Energy-Casimir functionals are used in classical works on nonlinear stability of Euler stationary flows [45, 46], and have been used to show the nonlinear stability of some of RSM equilibrium states [14, 47]).

Another class of variational problems (SFVP), that involve the stream function only (and not the vorticity), has been considered in relation with the RSM theory

$$
D(G)=\inf _{\psi}\left\{\int_{\mathscr{D}} d^{2} x\left[-\frac{1}{2}|\nabla \psi|^{2}+G(\psi)\right]\right\}(\mathrm{SFVP})
$$

Such (SFVP) functionals have been used to prove the existence of solutions to the equation describing critical points of (MVP) [47]. Interestingly, for the Quasi-geostrophic model, in the limit of small Rossby deformation radius, such a SFVP functional is similar to the Van-Der-Walls Cahn Hilliard model which describes phase coexistence in usual thermodynamics [17, 48]. This physical analogy has been used to make precise predictions in order to model Jovian vortices [17, 49]. (SFVP) functionals are much more regular than (CVP) functionals and thus also very interesting for mathematical purposes.

When we prescribe appropriate relations between the distribution function $d$, the functions $s$ and $G$, the three previous variational problems have the same critical points. This has been one of the motivations for their use in previous works. However, a clear description of the relations between the stability of these critical points is still missing (Is a (CVP) minimizer an RSM equilibria? Or does an RSM equilibria minimize (CVP)?). This has led to fuzzy discussions in recent papers. Providing an answer is a very important theoretical issue because, as explained previously, it leads to deep mathematical simplifications and will provide useful physical analogies.

In [42] we establish the relation between these three variational problems. The result is that any minimizer (global or local) of (SFVP) minimizes (CVP) and that any minimizer 
of (CVP) is an RSM equilibria. The opposite statements are wrong in general. For instance (CVP) minimizers may not minimize (SFVP), but may be instead only saddles. Similarly, RSM equilibria may not minimize (CVP) but be only saddles, even if no explicit example has yet been exhibited.

These results have several interesting consequences :

1. As the ensemble of (CVP) minimizers is a sub-ensemble of the ensemble of RSM equilibria, one can not claim that (CVP) are more relevant for applications than RSM equilibria.

2. The link between (CVP) and RSM equilibria provides a further justification for studying (CVP).

3. Based on statistical mechanics arguments, when looking at the Euler evolution at a coarse-grained level, it may be natural to expect the RSM entropy to increase. There is however no reason to expect such a property to be true for the Casimir's functional. As explained above, it may also happen that entropy extrema be (CVP) saddles.

\subsection{Classification of phase transitions}

Beyond the full characterization of ensemble inequivalence we have described above, there are many other qualitative features of the thermodynamics that depend only on the structure of the variational problems (5) and (6). Indeed, although the precise form of the solution obviously depends on the problem at hand through the functions $s(m)$ and $h(m)$, it is possible to classify all the different phenomenologies that one may find in the study of a particular long range interacting system. The questions in that respect are, increasing complexity at each step:

- what are the different possible types of generic points on an entropy curve $S(E)$ (these correspond to different phases)?

- what are the possible singular points of a generic $S(E)$ curve (these correspond to phase transitions)?

- what are the possible singular points on the $S(E)$ curve, when an external parameter is varied in addition to the energy (that is how phase transitions evolve when a parameter is varied)?

We address these different levels in the following paragraphs, using results from [37, 26]. These results are obtained by adapting to the dual variational problems (5) and (6) ideas that lead to the Landau classification of phase transitions. In the long range case however, there is no approximation involved, so the classification does not suffer from the problems of standard Landau theory (wrong critical exponents for instance). 


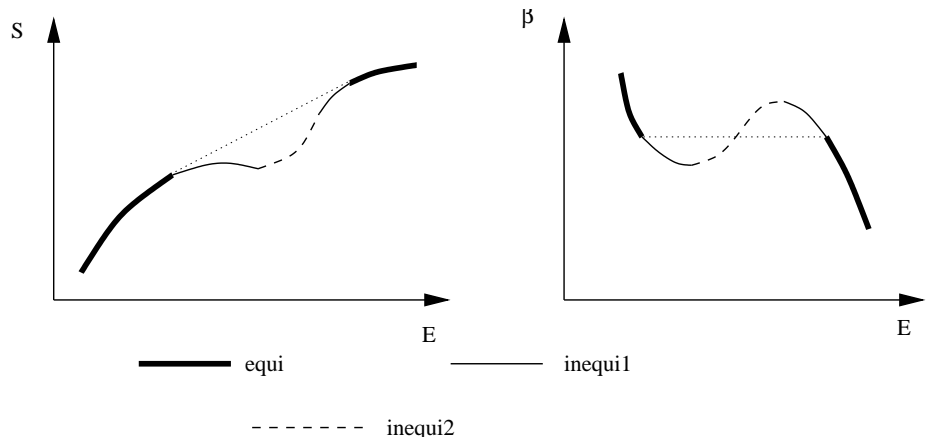

FIGURE 3. The three types of generic points; on the left: entropy $S(E)$ curve, on the right: caloric $\beta(E)$ curve. Thick, thin and dashed lines correspond respectively to the three types of points. The dotted lines shows the Maxwell construction giving the canonical solution in the inequivalence range.
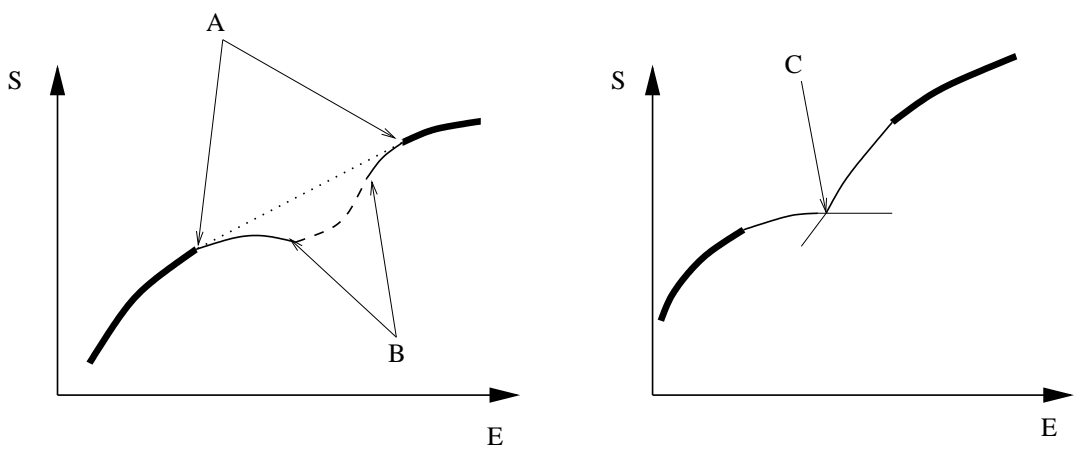

FIGURE 4. The three types of phase transitions (Codimension 0 singularities), for a system with no symmetry. A: canonical 1st order transition. B: canonical destabilization (a local minimum of (6) becomes a saddle point). C: microcanonical 1st order, temperature jump.

\subsubsection{Generic points of an entropy curve}

There are three types of generic points on the entropy curve, see Fig. 3:

- Concave points (that is $C_{v}>0$ ) where canonical and microcanonical ensembles are equivalent.

- Concave points where ensembles are not equivalent.

- Convex points $\left(C_{v}<0\right)$, where ensembles are always inequivalent.

\subsubsection{Singular points of a generic entropy curve: phase transitions}

Generic points as described above define segments of entropy curves, separated by singular points, that can be of several types. These points for systems without symmetry are classified in Fig. 4 . 


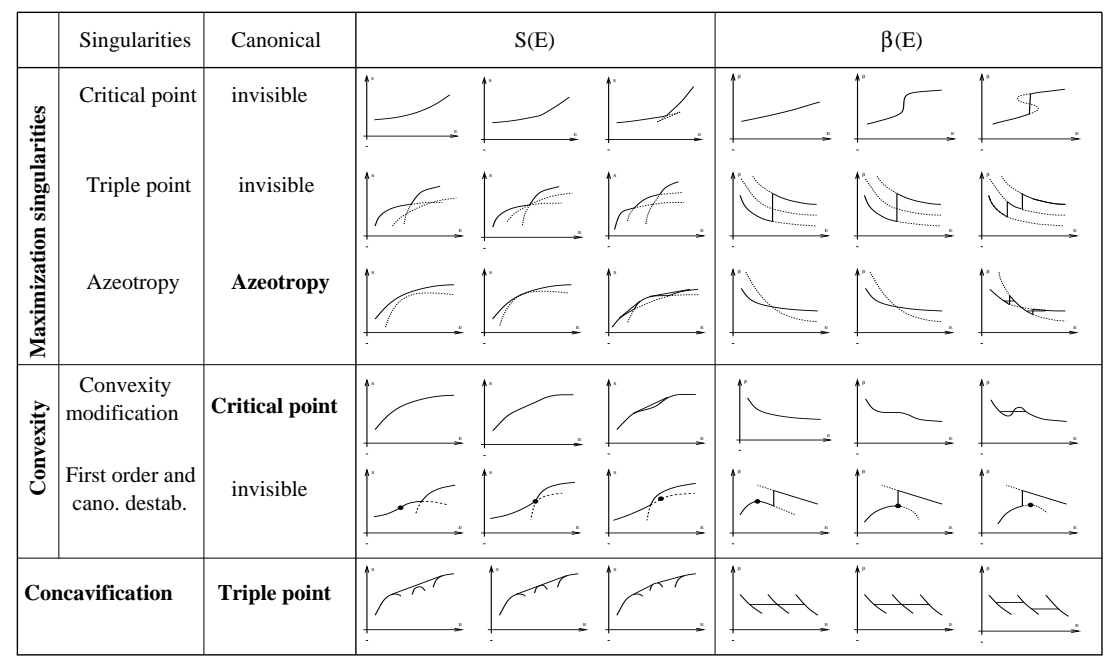

FIGURE 5. Classification of singularities, when one external parameter is varied (codimension 1 singularities). The first two columns give the singularity origin and name; the third one gives its status in the canonical ensemble: invisible means it has no consequence on the canonical solution; the six following plots are entropic and caloric curves showing the crossing of the singularity when the external parameter is varied. One recovers the usual phase transitions (triple point, azeotropy, critical point) in both ensembles. What is new is the list of the possible links between the behaviors in each ensemble, and the associated appearance of ensemble inequivalence. Please see [26] for a more detailed explanation, and singularities associated with symmetry breaking.

\subsubsection{Singular points on a singular entropy curve}

When an external parameter is varied, the entropy curve is modified. Some special values of the parameter correspond to qualitative changes for the phase transitions. All these possible qualitative changes are classified in [26]; Fig. 5] summarizes the results.

All types of phase transitions and ensemble inequivalences found in the literature so far are reproduced in the classification. In addition, the classification predicts the possibility of new phenomenologies, and new routes to ensemble inequivalence, that have not so far been observed in any specific model.

\subsection{Examples of new phase transitions}

In the previous section we explained the existence of many yet unobserved appearance of ensemble inequivalence, associated to phase transitions, as described in [26]. More recently the new finding of two such examples have been reported [50]: bicritical points (a bifurcation from a first order phase transition towards two second order phase transitions) and second order azeotropy (the simultaneous appearance of two second order phase transitions at a bifurcation). We present here these new phase transitions; they are associated with equilibrium states of the two dimensional Euler equation, when there is a linear relation between vorticity and stream function. This was first studied by Chavanis and Sommeria [51] in the context of the Robert-Sommeria-Miller (RSM) 
statistical mechanics of 2D flows [15, 14]. They found a criterion for the existence of a transition from a monopole to a dipole above a critical energy, for all (closed) domain geometry. In this section, we present an alternative method providing the same criterion, which generalizes to a large class of models, and thus shows the universality of the phenomenon. More interestingly this new method clarifies the nature of the phase transitions involved in this problem and makes the link with the existence of an ensemble inequivalence region. Those results are presented in a more general context in [50], where we note the interest of these phase transitions for very simple ocean models.

Euler equation and associated variational problem. Let us consider the 2D Euler equation in a closed domain $\mathscr{D}$. It can be written as a transport equation for the vorticity $\omega=\Delta \psi: \partial_{t} \omega+\mathbf{u} . \nabla \omega=0$. The velocity field $\mathbf{u}$ is related to $\omega$ via the stream function $\psi$ : $\mathbf{u}=\mathbf{e}_{z} \times \nabla \psi$, with $\psi=0$ on $\partial \mathscr{D}$. We introduce the projections $\omega_{i}$ of the vorticity $\omega$ on a complete orthonormal basis of eigenfunctions $e_{i}(x, y)$ of the Laplacian: $\Delta e_{i}=\lambda_{i} e_{i}$, where the $\lambda_{i}$ (all negative) are in decreasing order. The stationary states of this equation are prescribed by a functional relation $\omega=f(\psi)$. In the following we consider the solutions of the variational problem:

$$
S(E, \Gamma)=\max _{\omega}\{\mathscr{S}[\omega] \mid \mathscr{E}[\omega]=E \& \mathscr{C}[\omega]=\Gamma\}
$$

The variational problem (7) is similar to the generic problem (5) studied above, with two constraints instead of one.

- $\mathscr{S}$ is the entropy of the vorticity field $\omega$; we restrict ourselves to a quadratic functional: $\mathscr{S}[\omega]=-\frac{1}{2}\left\langle\omega^{2}\right\rangle_{\mathscr{D}}=-\frac{1}{2} \sum \omega_{i}^{2}$.

- $\mathscr{E}$ is the total energy: $\mathscr{E}[\omega]=\frac{1}{2}\left\langle(\nabla \psi)^{2}\right\rangle \mathscr{D}=-\frac{1}{2} \sum_{i} \lambda_{i} \omega_{i}^{2}$

- $\mathscr{C}$ is the circulation: $\mathscr{C}[\omega]=\langle\omega\rangle_{\mathscr{D}}=\sum_{i}\left\langle e_{i}\right\rangle \omega_{i}$ where $\left\langle e_{i}\right\rangle=\int_{\mathscr{D}} e_{i}(x, y) d x d y$

To compute critical points of the variational problem (7), we introduce two Lagrange parameters $\beta$ and $\gamma$, associated respectively with the energy and the circulation conservation. Those critical points are stationary solutions for the initial transport equation with $f(\psi)=\beta \psi-\gamma$. The solutions of the variational problem will thus provide the equilibrium states of the Euler equation that present a linear relationship between vorticity and stream function, for a given energy and circulation.

The aim of the following paragraphs is to determine which ones among the critical points are solutions of (7). It will then be possible to draw a phase diagram in the plane $(\Gamma, E)$ for those equilibrium states.

Dual quadratic variational problems. The problem (7), with two constraints, will be referred to as the microcanonical problem. As already explained earlier, it is sufficient to study the easier unconstrained ensembles, unless there is inequivalence of ensembles. The strategy is then the following. Start with the easiest problem:

$J(\beta, \gamma)=\min _{q}\{-\mathscr{S}[\omega]+\beta \mathscr{E}[\omega]+\gamma \mathscr{C}[\omega]\}$ (grand canonical). Check if all possible values of $E$ or $\Gamma$ correspond to a grand canonical solution; if yes the problem is solved, otherwise, we turn to the more constrained problem: $F(\beta, \Gamma)=\min _{q}\{-\mathscr{S}[q]+\beta \mathscr{E}[q] \mid \mathscr{C}[q]=\Gamma\}$ (canonical). 
In principle we could eventually have to solve the microcanonical problem. However, in this case, we will see that the microcanonical ensemble is equivalent to the canonical one: the whole range of $E$ and $\Gamma$ will be covered by canonical solutions.

We notice first that $\mathscr{S}, \mathscr{E}$ are quadratic functionals and that $\mathscr{C}$ is a linear functional.

We will thus have to look for the minimum of a quadratic functional with a linear part. Let us call $Q$ the purely quadratic part and $L$ the linear part of this functional. Then we have three cases

1. The smallest eigenvalue of $Q$ is strictly positive. The minimum exists and is achieved by a unique minimizer.

2. At least one eigenvalue of $Q$ is strictly negative. There is no minimum.

3. The smallest eigenvalue of $Q$ is zero (with eigenfunction $e_{0}$ ). If $L e_{0}=0$ (case $3 \mathrm{a}$ ), the minimum exists and each state of the neutral direction $\left\{\alpha e_{0}\right\}$ is a minimizer. If $L e_{0} \neq 0$, (case $3 b$ ) then no minimum exists.

The grand canonical ensemble. In that case the quadratic operator $Q$ associated to $\mathscr{J}=-\mathscr{S}+\beta \mathscr{E}+\gamma \mathscr{C}$ is diagonal in the Laplacian eigenvector basis. The variational problem admits a unique solution if and only if $\beta>\lambda_{1}$ (case 1 . above). If $\beta=\lambda_{1}$ (case 3. above), a neutral direction exists if and only if $\gamma=0$. By computing the energy and circulation of all those states, we prove that there is a unique solution at each point in the diagram $(E, \Gamma)$, below a parabola $\mathscr{P}$ (see figures 6 and 7 -a). Because the values of energies above the parabola $\mathscr{P}$ are not reached, we conclude that there is ensemble inequivalence for parameters in this region. We then turn to the more constrained canonical problem to find solutions in this area.

The canonical ensemble. The circulation is now fixed. We first transform this problem into an unconstrained variational problem. By using the circulation constraint, we express one coordinate in term of the others: $\omega_{1}=\left(\Gamma-\sum_{i} \omega_{i}\left\langle e_{i}\right\rangle\right) /\left\langle e_{1}\right\rangle$. This expression is then injected in the functional $\mathscr{F}=-\mathscr{S}+\beta \mathscr{E}$. The problem is now to find a minimizer $\left\{\omega_{i}\right\}_{i \geq 2}$ of this functional, without constraints. This case requires more computations that the previous one since the operator $Q$ associated to the quadratic part of $\mathscr{F}$ is no more diagonal in the basis $\left\{e_{i}\right\}$.

We first notice that if the domain geometry admits one or more symmetries, it generically exists eigenfunctions having the property $\left\langle e_{i}\right\rangle=0$. In the subspace spanned by all those eigenfunctions, $Q$ is diagonal, and its smallest eigenvalue is positive as long as $\beta>\beta_{1}^{0}$, where $\beta_{1}^{0}$ is the greatest $\lambda_{i}$ on this subspace. Then we look for the value of $\beta$ such that the smallest eigenvalue of $Q$ is zero in the subspace spanned by eigenfunctions with $\left\langle e_{i}\right\rangle \neq 0$. Let us call $\beta^{*}$ this value, and $\omega^{*}$ the corresponding eigenfunction: $Q\left[\omega^{*}\right]=0$. We find after some manipulation that $\beta^{*}$ is the greatest zero of the function $f(x)=1-x \sum_{i>1}\left\langle e_{i}\right\rangle^{2} /\left(x-\lambda_{i}\right)$. We conclude that there is a single solution to the variational problem if and only if $\beta>\max \left(\beta_{1}^{0}, \beta^{*}\right)$. When $\beta=\max \left(\beta_{1}^{0}, \beta^{*}\right)$, we distinguish two cases according to the sign of $\beta_{1}^{0}-\beta^{*}$ to discuss the existence of a neutral direction:

- i) $\beta_{1}^{0}<\beta^{*}$ we then consider $\beta=\beta^{*}$. There is a solution (case 3a) if $\Gamma=0$ and no solution (case $3 b$ ) for $\Gamma \neq 0$.

- ii) $\beta_{1}^{0}>\beta^{*}$ we then consider $\beta=\beta_{1}^{0}$. There is a solution (case 3 a) for all values of $\Gamma$. 

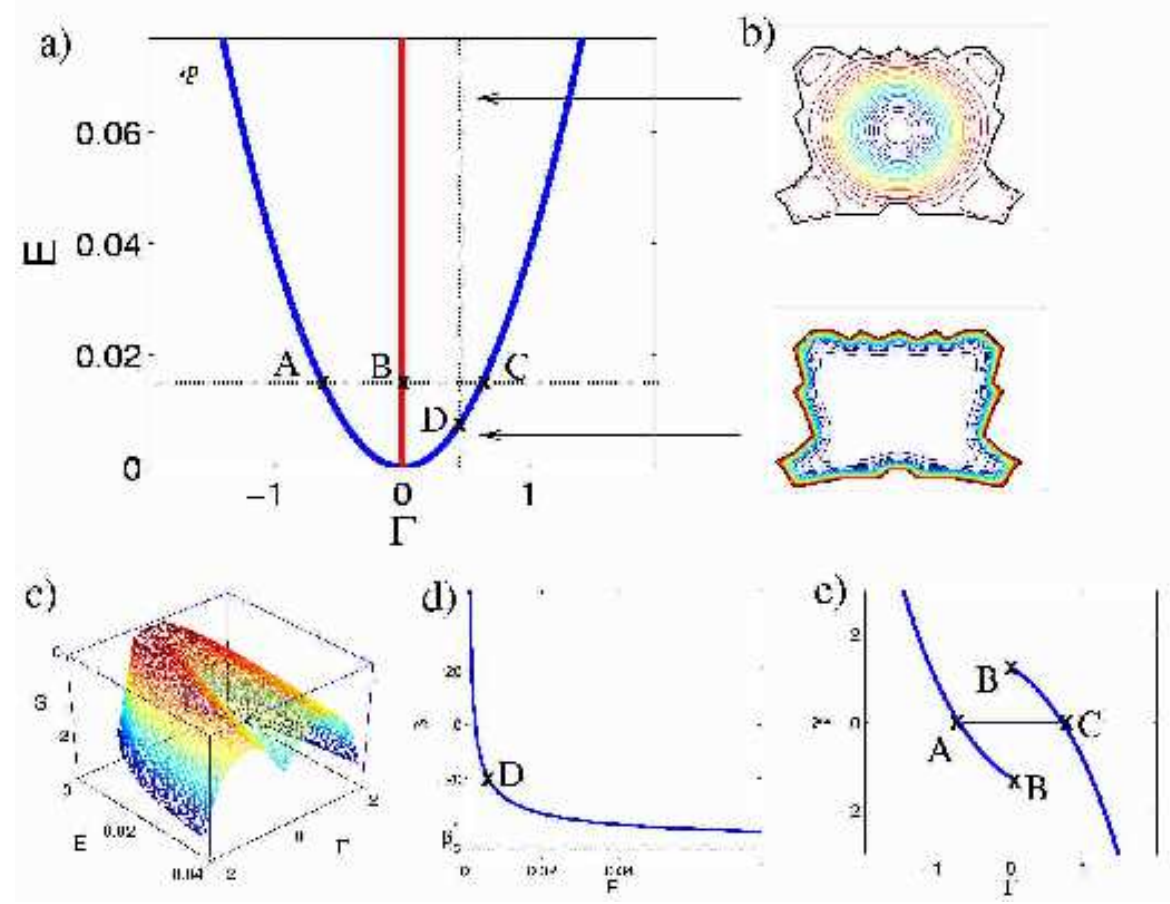

FIGURE 6. Case i) Phase diagram for a domain having no symmetry, or with a symmetry axis in the case $\beta^{*}<\beta_{1}^{0}$. a) Internal parameters are circulation $\Gamma$ and energy $E$. Straight line: first order transition. Parabola $\mathscr{P}$ : above this line there is no grand canonical solutions; only canonical and microcanonical solutions exist. It corresponds to an ensemble inequivalence region. b) examples of flow. c) Equilibrium entropy $S$ as a function of $E$ and $\Gamma$. We see clearly a region where the concave envelop of $S(E, \Gamma)$ is not equal to $S(E, \Gamma)$, which includes the first order transition line; d) $\beta=\partial S / \partial E$ at fixed $\Gamma$ : there is no singularity. e) $\gamma=\partial S / \partial \Gamma$ at fixed $E$ : there is a discontinuity of $\gamma$ at $\Gamma=0$ (first order transition). The Maxwell construction shows that the ensemble inequivalence region is associated with a first order transition in the ensemble where the value of the energy is fixed.

We thus obtain a criterion, namely the sign of $\beta_{1}^{0}-\beta^{*}$, that provides two classes of phase diagrams. This criterion depends only on the domain geometry. If the domain admits a symmetry axis, and is sufficiently stretched in a direction perpendicular to this axis, then $\beta_{1}^{0}>\beta^{*}$. For an ellipse, this is always the case. However, there are domains with a symmetry axis for which one can find a critical aspect ratio $\tau_{c}$ that separates the two cases. This is for instance the case of rectangular domains. For $\tau>\tau_{c}$, case ii) is realized $\left(\beta_{1}^{0}>\beta^{*}\right)$ and for $\tau<\tau_{c}$, case $\left.\mathrm{i}\right)$ is realized $\left(\beta_{1}^{0}<\beta^{*}\right)$. To conclude, we find two classes of phase diagrams:

- i) Domain without symmetry, or with symmetry with aspect ratio $\tau<\tau_{c}$ (see figure 6). If $\Gamma \neq 0$, all energy values are reached in the canonical ensemble, and there is a single solution for each point $(\Gamma, E)$. If $\Gamma=0$ (straight line on figure 6), two states coexist for each energy value $E$, and $\beta=\beta^{*}$.

- ii) Domain with symmetry and with an aspect ratio $\tau>\tau_{c}$ (see figure 7). There is a unique canonical solution at each point of the diagram below a parabola $\mathscr{P}_{0}$ in the plane $(E, \Gamma)$ (see the dashed line figure 7 ). Above this parabola, $\beta=\beta_{1}^{0}$, and there is 

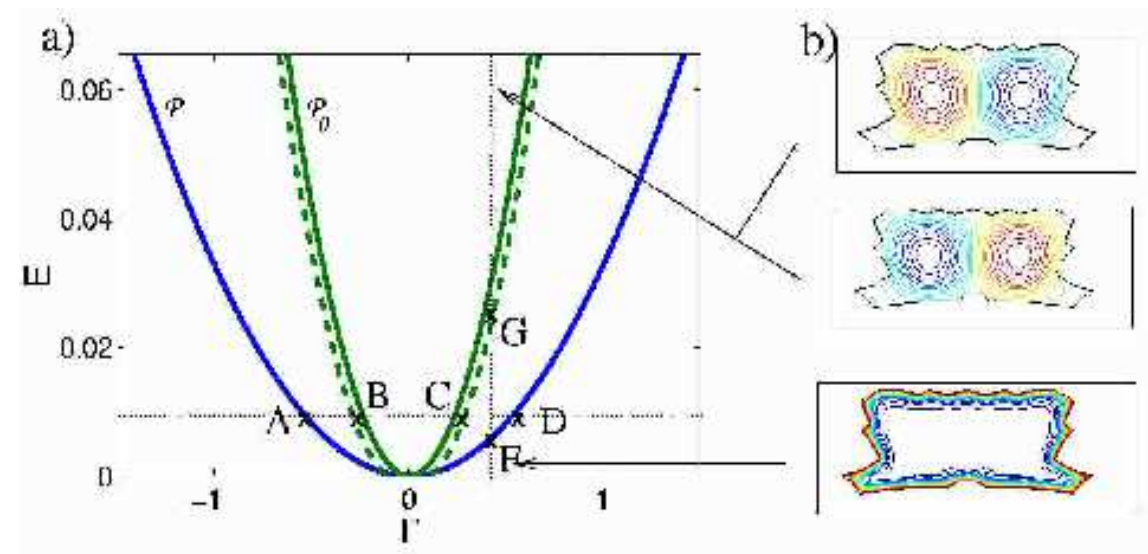

c)

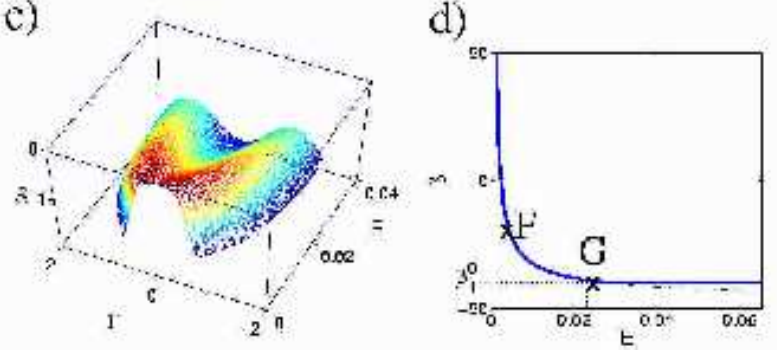

c)

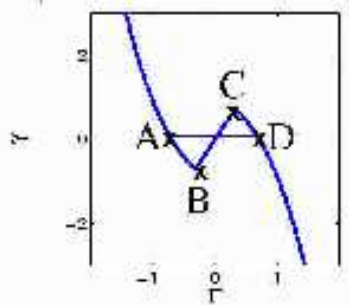

FIGURE 7. Case ii) Phase diagram for a domain having a symmetry axis, and sufficiently stretched in a direction perpendicular to this axis (case $\beta^{*}>\beta_{1}^{0}$ ). a),b),c),d),e): see figure (6). Dashed parabola $\mathscr{P}_{0}$ : second order transition line. Above this line, $\partial S / \partial E=\beta_{1}^{0}$, and the choice of one state among two possibilities breaks the system's symmetry. We see that $\partial \beta / \partial E$ is discontinuous at point $G$, as well as $\partial \gamma / \partial \Gamma$ at points $B$ and $C$, which is the signature of a second order phase transition.

two canonical solutions at each point of the diagram. They differ only by the sign of the contribution of the Laplacian eigenvector associated to the eigenvalue $\beta_{1}^{0}$ (it is a dipole). Below the Parabola, this eigenmode has no contribution to the solution. The choice of one solution among the two possibilities breaks the system's symmetry when $\mathscr{P}_{0}$ is crossed. At high energy, the contribution of the dipole is dominant.

In both cases we find that all circulation and energy values have been reached by canonical solutions. We conclude that microcanonical and canonical ensembles are equivalent: all microcanonical solutions are also canonical solutions.

Description of phase transitions. The difference between the two classes of flow is the existence of either first or second order phase transitions, corresponding respectively to case i) and ii). The main observation is that first order and second order microcanonical transitions always take place in the ensemble inequivalence area. In that respect, those transitions are signatures of ensemble inequivalence for this class of twodimensional flows, which could be observed in laboratory experiments on quasi two dimensional flows.

The transition from systems of type i) to systems of type ii), when the geometry is modified, leads to one of the predicted, but yet unobserved phase transition of the classification [26], described here in section Classification of Phase Transitions. On 


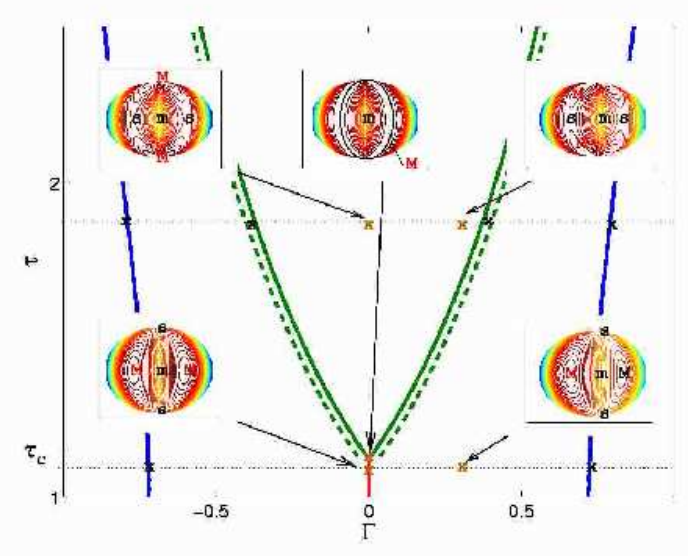

FIGURE 8. Bicritical point (from Ref. [50]) for a rectangular domain with aspect ratio $\tau$. Bifurcation from a first order transition line to two second order phase transition lines. Insets are schematic projections of the Entropy $\mathscr{S}[\omega]$ in a plane $\left(\omega_{1}^{0}, \omega^{*}\right)$, by taking into account the constraints. M: maximum. m: minimum. s: saddle.

figure 8, we consider a fixed energy, and present the phase diagram in the $(\Gamma, \tau)$ plane, where $\tau$ is an external parameter characterizing the aspect ratio of the domain. In the microcanonical ensemble, there is a bifurcation from a first order transition line to two second order transition lines at a critical value $\tau=\tau_{c}$. Such a bifurcation is referred to as a bicritical point.

\subsection{Challenges in the equilibrium statistical mechanics}

It seems fair to say that the equilibrium statistical mechanics of long range interacting systems is well understood at a fundamental level, despite the important differences with the short range case. However, some challenges and questions remain open; we mention here some of them.

A first issue is the relevance for natural phenomenon or laboratory experiments: is it possible to identify situations where equilibrium statistical mechanics satisfactorily describes the structures observed (like in Jovian atmosphere [17])?

The main challenge is probably to observe negative specific heat and ensemble inequivalence in experiments or in real physical systems. Up to now, this has not been possible. A first possibility would be to observe natural phenomena corresponding to statistical microcanonical equilibria, which are characterized by negative specific heat, like for instance geophysical flows in microcanonical situations. Another possibility would be to achieve this in the lab. One should consider a physical system sufficiently simple in order to be able to characterize ensemble inequivalence, or the computation of negative specific heat. Moreover, exchanges of energy with the environment have to be negligible over a sufficiently long time, in order to make sure that the microcanonical ensemble is the relevant one; this imposes severe constraints on any laboratory setup. Several candidates have been considered. One of the most popular one may be to build synthetic 
magnetic systems with long range interactions. Another possibility could be to design simple two dimensional flow experiments or two dimensional plasma experiments, in order to reproduce the recently predicted ensemble inequivalence, as briefly describe in [50].

There also several theoretical issues :

- Could we find physical examples of the new phase transitions found in the classification?

- The structure of the dual variational problems (5) and (6) appears in other physical contexts. Could the results described here, like for instance the classification of phase transitions, have an interest, when applied to these different situations? For a step in this direction, see for instance [52].

- A very interesting and difficult challenge would be to make the classification [26] rigorous. This implies to give a precise mathematical definition to the notion of a normal form for a mean field variational problem.

\section{KINETIC THEORIES OF SYSTEMS WITH LONG RANGE INTERACTIONS}

The previous section provided a brief summary of old and new results for long range interacting systems at equilibrium. Unfortunately, it turns out that the coherent structures these systems form, and the stationary states they reach are generally out of equilibrium. Although knowledge of equilibrium is a useful benchmark, which usually yields a qualitative understanding of the physics, some new techniques are needed to really understand the phenomena at hand. Clearly, we have to reintroduce the time in our framework and study the dynamics of the systems.

We have seen that for systems with long range interactions, a mean field approach is usually exact in the limit of a large number of particles, when one wants to describe the equilibrium macrostates. This is valid thanks to an averaging of the potential over many particles. In the following we explain that a similar mean field approach is also valid for the dynamics: at each time the potential and the force can be expressed with a very good approximation from the one particle distribution function, and thus the BBGKY hierarchy can be safely truncated.

This well understood fact is the base of the kinetic theory for the dynamics of systems with long range interaction. This led to the classical kinetic theories of self gravitating stars, plasmas in the weak coupling limit, or point vortex models in two dimensional turbulence. In the limit of a large number of particles, such dynamics is well approximated by kinetic theories [53, 54], 16, 55, 56, 57]: to leading order in $1 / \sqrt{N}$ the dynamics is of a Vlasov type; after a much longer time, the relaxation towards equilibrium is governed by Lenard-Balescu type dynamics (or its approximation by the Landau equation).

In the next subsection, we introduce briefly the Vlasov dynamics, and the issue of its time of validity. For a large number $N$ of particles, these systems may exhibit quasistationary states (QSS) [58, 59] (in the plasma or astrophysical context see for instance [60, 53]). We give a kinetic interpretation of such states as stable stationary solutions of 
the Vlasov dynamics.

An interesting question is whether we can predict such Quasi-Stationary States, from the initial condition of the Vlasov equation, using statistical mechanics. In the following subsection, we present recent studies on the equilibrium statistical mechanics of the Vlasov equation (and not of the $\mathrm{N}$ particle dynamics) [61, 62, 63], in the spirit of LyndenBell's work [1] in the context of self-gravitating stars.

We then turn to the kinetic theory of these systems beyond the time of validity of Vlasov equation, which leads to the Lenard-Balescu equation. This allows to address the important question of the time scale for the relaxation to equilibrium: this time scale may be of order $N / \log N$ (this is a classical result by Chandrasekhar for relaxation to equilibrium of self-gravitating stars, or of a plasma), of order $N$ (for a smooth potential), or much larger than $N$ (this is related to the recent result that the Lenard-Balescu operator identically vanishes for one dimensional systems [55]). This last result explains the striking numerical observation of an $N^{1.7}$ time scale in the HMF model [59].

In the following subsection, we explain how a classical kinetic approach allows to describe the stochastic process of a single particle in a bath composed by a large number of other particles. This stochastic process is governed by a usual Fokker-Planck equation. In classical papers, this bath is at equilibrium. We stress here that this bath can also be a bath of particles in an out of equilibrium Quasi Stationary State. We explain recent new results [55] proving that this Fokker Planck equation has no spectral gap, and lead to long time algebraic correlations and anomalous diffusion. This provides a quantitative prediction for the algebraic autocorrelation function and anomalous diffusion indices, previously observed in some numerical computations [64, 65, 66]. These theoretical predictions have been numerically checked in [67]. Some more recent related results have also been reported in [68]. We note that an alternative explanation, both for the existence of QSS and for anomalous diffusion has been proposed in the context of Tsallis non extensive statistical mechanics [69, 66] (see [59] and [55, 70] for further discussions).

The last subsection is devoted to describe some remaining issues and challenges in the context of the classical kinetic theory for systems with long range interactions. We also note that we do not describe many other existing dynamical properties which are common to systems with long range interactions: vanishing Lyapounov exponents [58, 71], breaking of ergodicity [72, 73, 74], ans so on. All of the common dynamical properties of systems with long range interactions are a result of similar collective (selfconsistent) dynamics [75].

\subsection{Vlasov dynamics and Quasi-Stationary states}

As for the equilibrium statistical mechanics, one needs to choose a scaling to study the kinetic theory; again, the scaling described in the equilibrium context, which ensures that each particle experiences a force of order 1, is the appropriate one. The goal is now to approximate the dynamics of $N$ ordinary differential equations for the discrete particles dynamics by a single Partial differential equation for the one-particle distribution function. 
For definiteness, we consider the following Hamiltonian system:

$$
\left\{\begin{array}{l}
\dot{x}_{i}=p_{i} \\
\dot{p}_{i}=-\frac{1}{N} \sum_{j \neq i} \frac{d V}{d x}\left(x_{i}-x_{j}\right)
\end{array}\right.
$$

The range of the potential $V$ is supposed to be of the same order of magnitude as the total size of the system: this is our definition for "long range interaction"1.

Consider the following continuous approximation of the potential:

$$
\Phi(x, t)=\int V(y-x) f(y, p, t) d y d p
$$

and the corresponding equation for the one-particle distribution function $f$ (this is the Vlasov equation):

$$
\frac{\partial f}{\partial t}+p \frac{\partial f}{\partial x}-\frac{\partial \Phi}{\partial x} \frac{\partial f}{\partial p}=0
$$

Replacing the true discrete potential by $\Phi$ neglects correlations between particles and finite- $N$ effects. However, as each particle interacts at any time with an extensive number of other particles, one may hope that this mean field approach correctly reproduces the potential experienced by a particle, and becomes exact in the infinite $N$ limit. Under some regularity assumptions for the potential $V$, this is indeed correct, and it has been rigorously proved (see [76] for a very regular $V$, [77] for a mildly singular potential). To be more precise, these theorems state the following: take a discrete $N$-particles initial condition and an initial continuous one-particle distribution function $f(x, p, 0)$ which is close, in some sense, to the former; evolve the $N$ particles according to (8), and evolve $f(x, p, 0)$ according to (9) and (10); then the $N$-particles dynamics and $f(x, p, t)$ will remain close for a time at least of the order of $\log N$. Several remarks are in order:

1. This implies that if the $t \rightarrow \infty$ limit is taken for a fixed $N$, finite- $N$ effects will come into play; the evolution will then depart from the Vlasov equation, and we expect the system to eventually reach the statistical equilibrium. However, for any finite time $T$ there exists some $N$ such that the system approximately follows the Vlasov equation up to time $T$.

2. The $\log N$ is optimal in the sense that there exist initial conditions such that the discrete (8) and Vlasov (10) dynamics diverge on such a time scale (see [78] for further discussion). ${ }^{2}$

3. However, this "coincidence time" may in some cases be much longer: for instance, discrete initial conditions close to a stable stationary state of the Vlasov equation stay so for a time algebraic in $N$ (see [59] for a numerical observation and [80] for a mathematical investigation of the phenomenon).

\footnotetext{
1 Albeit rather general, equations (8) do not include the 2D flows, nor the wave-particles models; most of the following discussion does apply to these cases too, with small modifications.

2 A recent consideration of the thermodynamic stability of a mean field Ising model with stochastic dynamics has found the relaxation time to be logarithmic in $N$ [79].
} 
4. The analogous result for $2 \mathrm{D}$ flows is the convergence of the dynamics of discrete vortices to the corresponding continuous partial differential equation for the vorticity field (Euler, Quasi-geostrophic...). For 2D flows however, the fundamental equation is the PDE, contrary to the classical particles case. A mathematical proof of convergence is given in [81]. For wave-particles systems, the analogous theorem is given in [82].

5. The mathematical proofs cited above do not include the gravitational and electrostatic $1 / r$ cases. It seems however reasonable to believe that some convergence result towards the Vlasov equation still holds in this case; Vlasov equation is routinely used by physicists for these potentials.

In the light of the previous remarks, and if the number of particles $N$ is big enough, the following dynamical scenario now seems reasonable:

- Starting from some initial condition, the $N$-particles system approximately follows the Vlasov dynamics, and evolves on a time scale of order 1.

- It then approaches a stable stationary state of the Vlasov equation; the Vlasov evolution stops.

- Because of discreteness effects, the system evolves on a time scale of order $N^{\alpha}$ for some $\alpha$, and slowly approaches the full statistical equilibrium, moving along a series of stable stationary states of the Vlasov equation.

In this scenario, the $N$-particles system gets trapped for long times out of equilibrium, close to stable stationary states of the Vlasov equation: these are then called "quasi stationary states" in the literature. This is the basis for the "violent relaxation" theory of Lynden-Bell [1]; Refs. [61, 59, 83] give examples of this scenario for wave-particles, the HMF and astrophysical models. The next problem is then to study the stable stationary states of the Vlasov equation, that is the candidates for the "quasi stationary states". Before turning to this in the next paragraph, let us note that there is however no reason for this scenario to be the only possibility: for instance, the Vlasov dynamics may converge to a periodic solution of the Vlasov equation [84].

The Vlasov equation (as well as the Euler equation and its variants) has many invariants: beside the energy $H[f]$ (and possibly the linear or angular momentum), inherited from the discrete Hamiltonian equations, the following quantities $C_{s}[f]$, sometimes called Casimirs, are conserved for any function $s$ :

$$
C_{s}[f]=\int s(f(x, p, t)) d x d p .
$$

Using these invariants, it is possible to construct many stationary states of the Vlasov equation. Consider the following variational problem, for a concave function $s$ :

$$
\sup _{f}\left\{\int s(f(x, p)) d x d p \mid \int f d x d p=1, H[f]=e\right\} .
$$

Any solution of this variational problem yields a stationary solution of the Vlasov equation. In addition, the variational structure of the construction is very useful to study the stability of such states (see [29, 85] for more details). There is no constraint on the 
concave function $s$, so that we have very many stable stationary states of the Vlasov equation. As a consequence, many numerical or experimental results can be fitted with a good choice of $s$; this is also a serious limit of the theory: without a recipe to choose the right stationary state, the theory is not predictive. We address this problem in the next paragraph.

We have explained that any Vlasov stable stationary solution is a Quasi Stationary State. Then, because inhomogeneous Vlasov stationary states do exist, one should not expect Quasi Stationary States to be homogeneous. This is illustrated in the case of several generalizations of the HMF model in Ref. [78].

The issue of the robustness of QSS when the Hamiltonian is perturbed by short range interactions [86] or when the system is coupled to an external bath [87] has also been addressed, and it was found that while the power law behavior survives, the exponent may not be universal.

\subsection{Lynden Bell statistical mechanics}

Under the Vlasov dynamics, the distribution function $f$ is advected, by a field which itself depends on $f$. The conservation of Casimirs amounts to the conservation of the area of all level sets

$$
I_{[a, b]}=\{(x, p) \text { such that } a \leq f(x, p, t) \leq b\} ;
$$

as time goes by the sets are filamented down to a finer and finer scale, and the filaments get interwoven. Understanding the long time behavior of this complicated dynamics is not an easy task, analytically or numerically. Assuming that $f$ tends to one of the many stable stationary states of the Vlasov equation, the Lynden-Bell statistical mechanics is a recipe to choose the right one. In a nutshell, at fixed energy (and possibly linear or angular momentum), it selects the most mixed state compatible with the Casimirs conservation. It is a maximum entropy theory; the Lynden-Bell equilibrium is given by the solution of a problem like (12), the function $s$ being determined by probability theory and the initial distribution $f(x, p, t=0)^{3}$.

The idea goes back to a pioneering work of Lynden-Bell in the context of astrophysics in 1967 [1]; the problem was later revisited by Chavanis and collaborators [88], in connexion with the statistical mechanics of 2D flows. Let us note that the analog of Lynden-Bell theory for the Euler and Euler-like equations of 2D flows is the RobertSommeria-Miller theory [89, 15, 14]: it relies on the very same ideas.

The Lynden-Bell and Robert-Sommeria-Miller theories have had important successes; let us mention here the descriptions of the core of elliptical galaxies, and the giant vortices in Jupiter's atmosphere [17]. However, this is the exception rather than the rule. A lot of works have been devoted to checking these theories in different contexts, to which we do not do justice here. To summarize them very briefly, the rule is that

\footnotetext{
3 We have to mention that the determination of the Lynden-Bell equilibrium is in general a difficult task; the calculations are usually practical only for two- or three-levels initial distributions.
} 
the phase space mixing induced by the Vlasov equation is not strong enough, so that the theoretical predictions are in general at best qualitatively correct (see [63] for a discussion of these issues; see also [90]).

\subsection{Order parameter fluctuations and Lenard-Balescu equation}

In this section, we explain briefly how one classically obtains exact expressions for the $1 / \sqrt{N}$ fluctuations of the order parameter, for a system with long range interactions close to a Quasi Stationary State. In order to make this discussion as simple as possible, we treat the case of the HMF model, a one dimensional system with a smooth two body potential $V$. We follow [55], and refer to [91] for a plasma physics treatment, to [56, 57, 16] for the case of point vortices and to Ref. [92] for self-gravitating stars.

One could use an asymptotic expansion of the BBGKY hierarchy, where $1 / \sqrt{N}$ is the small parameter, and obtain the same results. The $1 / \sqrt{N}$ fluctuations would then have been obtained by explicitly solving the dynamical equation for the two point correlation function, while truncating the BBGKY hierarchy by assuming a Gaussian closure for the three point correlation function. Such a procedure is justified in the large $N$ limit (see Ref. [54]). Our presentation rather follows the Klimontovich approach.

The state of the $N$-particles system can be described by the discrete single particle time-dependent density function $f_{d}(t, x, p)=\frac{1}{N} \sum_{j=1}^{N} \delta\left(x-x_{j}(t)\right) \delta\left(p-p_{j}(t)\right)$, where $\delta$ is the Dirac function, $(x, p)$ the Eulerian coordinates of the phase space and $\left(x_{i}, p_{i}\right)$ the Lagrangian coordinates of the particles. The dynamics is thus described by the Klimontovich's equation [54].

$$
\frac{\partial f_{d}}{\partial t}+p \frac{\partial f_{d}}{\partial x}-\frac{d V}{d x} \frac{\partial f_{d}}{\partial p}=0
$$

where the potential $V$ that affects all particles is $V(t, x) \equiv-\int_{0}^{2 \pi} d y \int_{-\infty}^{+\infty} d p \cos (x-$ y) $f_{d}(t, y, p)$. This description of the Hamiltonian dynamics derived from (11) is exact : as the distribution is a sum of Dirac functions it contains the information on the position and velocity of all the particles. It is however too precise for usual physical quantities of interest but will be a key starting point for the derivation of approximate equations, valid in the large $N$ limit and describing average quantities.

When $N$ is large, it is natural to approximate the discrete density $f_{d}$ by a continuous one $f(t, x, p)$. Considering an ensemble of microscopic initial conditions close to the same initial macroscopic state, one defines the statistical average $\left\langle f_{d}\right\rangle=f_{0}(x, p)$, whereas fluctuations of probabilistic properties are of order $1 / \sqrt{N}$. We will assume that $f_{0}$ is any stable stationary solution of the Vlasov equation. The discrete time-dependent density function can thus be rewritten as $f_{d}(t, x, p)=f_{0}(x, p)+\delta f(t, x, p) / \sqrt{N}$, where the fluctuation $\delta f$ is of zero average. We define similarly the averaged potential $\langle V\rangle$ and its corresponding fluctuations $\delta V(t, x)$ so that $V(t, x)=\langle V\rangle+\delta V(t, x) / \sqrt{N}$. Inserting both expressions in Klimontovich's equation (13) and taking the average, one obtains

$$
\frac{\partial f_{0}}{\partial t}+p \frac{\partial f_{0}}{\partial x}-\frac{d\langle V\rangle}{d x} \frac{\partial f_{0}}{\partial p}=\frac{1}{N}\left\langle\frac{d \delta V}{d x} \frac{\partial \delta f}{\partial p}\right\rangle \text {. }
$$


The lhs is the Vlasov equation. The exact kinetic equation (14) suggests that the quasistationary states of sections 3.1 and 3.2 do not evolve on time scales much smaller than $N$; this would explain the extremely slow relaxation of the system towards the statistical equilibrium.

Let us now concentrate on stable homogeneous distributions $f_{0}(p)$, which are stationary since $\langle V\rangle=0$. Subtracting Eq. (14) from Eq. (13) and using $f_{d}=f_{0}+\delta f / \sqrt{N}$, one gets

$$
\frac{\partial \delta f}{\partial t}+p \frac{\partial \delta f}{\partial x}-\frac{d \delta V}{d x} \frac{\partial f_{0}}{\partial p}=\frac{1}{\sqrt{N}}\left[\frac{d \delta V}{d x} \frac{\partial \delta f}{\partial p}-\left\langle\frac{d \delta V}{d x} \frac{\partial \delta f}{\partial p}\right\rangle\right]
$$

For times much shorter than $\sqrt{N}$, we may drop the rhs encompassing quadratic terms in the fluctuations. The fluctuating part $\delta f$ are then described, by the linearized Vlasov equation (this is another result of the Braun and Hepp theorem [76, 93]). This suggests to introduce the spatio-temporal Fourier-Laplace transform of $\delta f$ and $\delta V$. This leads to

$$
\widetilde{\delta V}(\omega, k)=-\frac{\pi\left(\delta_{k, 1}+\delta_{k,-1}\right)}{\varepsilon(\omega, k)} \int_{-\infty}^{+\infty} d p \frac{\widetilde{\delta f}(0, k, p)}{i(p k-\omega)}
$$

where

$$
\varepsilon(\omega, k)=1+\pi k\left(\delta_{k, 1}+\delta_{k,-1}\right) \int_{-\infty}^{+\infty} d p \frac{\frac{\partial f_{0}}{\partial p}}{(p k-\omega)}
$$

is the dielectric permittivity. The evolution of the potential autocorrelation, can therefore be determined. For homogeneous states, by symmetry, $\left\langle\widetilde{\delta V}\left(\omega_{1}, k_{1}\right) \widetilde{\delta V}\left(\omega_{2}, k_{2}\right)\right\rangle=0$ except if $k_{1}=-k_{2}= \pm 1$.

\subsubsection{Autocorrelation of the potential}

One gets, after a transitory exponential decay, the general result

$$
\left\langle\delta V\left(t_{1}, \pm 1\right) \delta V\left(t_{2}, \mp 1\right)\right\rangle=\frac{\pi}{2} \int_{\mathscr{C}} d \omega e^{-i \omega\left(t_{1}-t_{2}\right)} \frac{f_{0}(\omega)}{|\varepsilon(\omega, 1)|^{2}}
$$

This is an exact result, no approximation has been done yet.

\subsubsection{Lenard Balescu equation}

A similar, but longer, calculation allows to compute the rhs. of Eq. (14), at order $1 / N$. This is very interesting as it gives access to the slow evolution of the distribution $f_{0}$ due to the "collisional" effects. This is, for systems with long range interactions, the analogue of the Boltzmann equation for dilute system with short range interactions. We do not describe the computation in details (see [91, 54]), as we just want to discuss 
qualitatively the collision operator. This collision operator is called the Lenard Balescu operator and it leads to the Lenard Balescu equation.

For system of particle with long range interactions given by a two body potential $\frac{1}{N} V\left(\mathbf{x}_{1}-\mathbf{x}_{2}\right)$, the Lenard Balescu equation reads :

$$
\frac{\partial f_{0}(\mathbf{p}, t)}{\partial t}=-\frac{1}{N} \frac{\partial}{\partial \mathbf{p}} \cdot\left[\int d \mathbf{k} d \mathbf{p}^{\prime} \frac{\phi(k)}{\left|\varepsilon\left(k, \mathbf{k} \cdot \mathbf{p}^{\prime}\right)\right|} \mathbf{k} \cdot\left(f_{0}(\mathbf{p}) \frac{\partial f_{0}}{\partial \mathbf{p}}\left(\mathbf{p}^{\prime}\right)-f_{0}\left(\mathbf{p}^{\prime}\right) \frac{\partial f_{0}}{\partial \mathbf{p}}(\mathbf{p})\right) \delta\left(\mathbf{k} \cdot\left(\mathbf{p}-\mathbf{p}^{\prime}\right)\right)\right],
$$

where $\mathbf{k}$ is a wave vector, $\phi(k)$ is the Fourier transform of the potential $V(\mathbf{x})$, and $\left|\varepsilon\left(k, \mathbf{k} \cdot \mathbf{p}^{\prime}\right)\right|$ is the dielectric permittivity. One note that this is a quadratic operator, as for the Boltzmann equation. Moreover, this operator involve a resonance condition in the Dirac distribution $\delta\left(\mathbf{k} .\left(\mathbf{p}-\mathbf{p}^{\prime}\right)\right)$.

$>$ From this equation one clearly expects a relaxation towards equilibrium of any Quasi-Stationary state with a characteristic time of order $N$. We note that for plasma or self gravitating systems, due to the small $r$ divergence of the interaction potential, the Lenard Balescu operator diverges at small scales. This is regularized by close two body encounters, fixing a small scale cutoff. This leads to a logarithmic correction to the relaxation time, which is then the Chandrasekhar time proportional to $\log (N) / N$.

One clearly sees on equation (18) that the mechanism for evolution of the distribution function is related to the resonances of two particles. An essential point is that the condition $\mathbf{k}$. $\left(\mathbf{p}-\mathbf{p}^{\prime}\right)=0$ cannot be fulfilled for one dimensionnal systems. It would indeed imply $p=p^{\prime}$, and because the Lenard Balescu operator is odd in the variable $p$, it will vanish. Another way to obtain the same result, is to directly compute the rhs of Eq. (14). We do not report such long and tedious computations, but it shows that it identically vanishes at order $1 / N$, for one dimensional systems.

This proves that Vlasov stable distribution function will not evolve on time scales smaller or equal to $N$. This is an important result: generic out of equilibrium distributions, for one dimensionnal systems, evolve on time scales much larger than $N$. This is in agreement with the $N^{1.7}$ scaling law which was numerically reported [59].
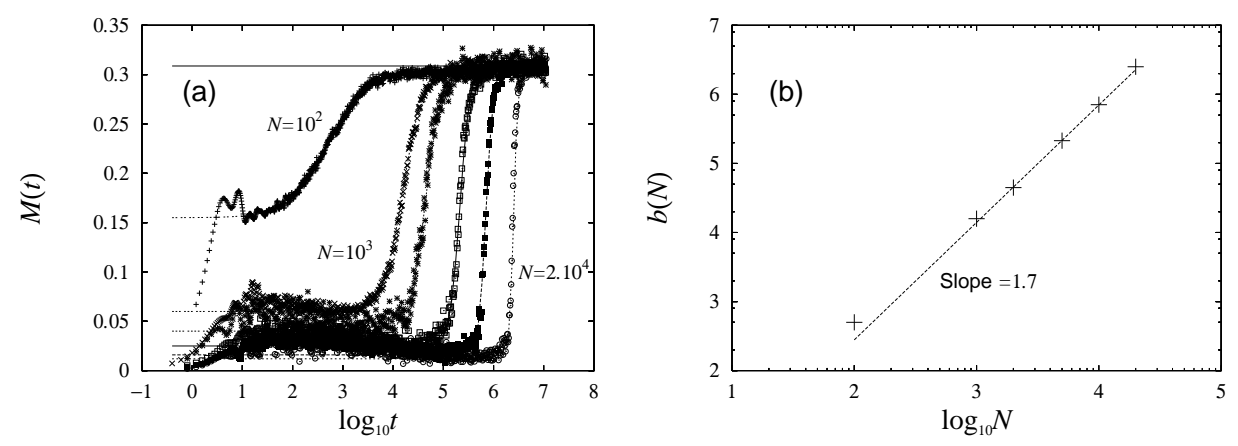

FIGURE 9. Panel (a) presents the temporal evolution of the magnetization $M(t)$, for the HMF model, for different particles numbers: $N=10^{2}\left(10^{3}\right), 10^{3}\left(10^{2}\right), 2.10^{3}(8), 5.10^{3}(8), 10^{4}(8)$ and $2.10^{4}(4)$ from left to right, the number between brackets corresponding to the number of samples. The horizontal line represents the equilibrium value of $M$. Panel (b) shows the logarithmic timescale $b(N)$ as a function of $N$, whereas the dashed line represents the law $10^{b(N)} \sim N^{1.7}$. From Ref. [59]. 


\subsubsection{The stochastic process of a single particle in a bath}

Let us now consider relaxation properties of a test-particle, indexed by 1 , surrounded by a background system of $(N-1)$ particles with a homogeneous distribution. The fluctuation of the potential is thus

$$
\delta V(t, x) \equiv-\int_{0}^{2 \pi} d y \int_{-\infty}^{+\infty} d p \cos (x-y) \delta f(t, y, p)-\frac{1}{\sqrt{N}} \cos \left(x-x_{1}\right) .
$$

Using the equations of motion of the test particle and omitting the index 1 for the sake of simplicity, one obtains $p(t)=p(0)-\int_{0}^{t} d u(d \delta V(u, x(u))) /(d x) / \sqrt{N}$. By introducing iteratively the expression of $x$ in the rhs and expanding the derivative of the potential, one gets the result at order $1 / N$. The key point is that this approach does not use the usual ballistic approximation. As a consequence, we obtain an exact result at order $1 / N$. This is of paramount importance here to treat accurately the collective effects. As the changes in the impulsion are small (of order $1 / \sqrt{N}$ ), the description of the impulsion stochastic process by a Fokker-Planck equation is valid. This last equation is then characterized by the time behavior of the first two moments $\left\langle(p(t)-p(0))^{n}\right\rangle$. Using the generalization of formula (17) when the effect of the test particle is taken into account, one obtains in the large $t$-limit

$$
\begin{aligned}
\langle(p(t)-p(0))\rangle & \underset{t \rightarrow+\infty}{\sim} \frac{t}{N}\left(\frac{d D}{d p}(p)+\frac{1}{f_{0}} \frac{\partial f_{0}}{\partial p} D(p)\right) \\
\left\langle(p(t)-p(0))^{2}\right\rangle & \underset{t \rightarrow+\infty}{\sim} \frac{2 t}{N} D(p),
\end{aligned}
$$

where the diffusion coefficient $D(p)$ can be written as

$$
D(p)=2 \operatorname{Re} \int_{0}^{+\infty} d t e^{i p t}\langle\delta V(t, 1) \delta V(0,-1)\rangle=\frac{\pi^{2} f_{0}(p)}{|\varepsilon(p, 1)|^{2}} .
$$

These results are the exact leading order terms in an expansion where $1 / N$ is the small parameter.

Using time variable $\tau=t / N$ as suggested by Eqs. (20) and (21), the Fokker-Planck equation describing the time evolution of the distribution of the test particle is

$$
\frac{\partial f_{1}(\tau, p)}{\partial \tau}=\frac{\partial}{\partial p}\left[D(p)\left(\frac{\partial f_{1}(\tau, p)}{\partial p}-\frac{1}{f_{0}} \frac{\partial f_{0}}{\partial p} f_{1}(\tau, p)\right)\right] .
$$

We stress that this equation depends on the bath distribution $f_{0}$. It is valid both for equilibrium and and out of equilibrium $f_{0}$, provided that $f_{0}$ is a stable stationary solution of the Vlasov equation. In the limit $\tau \rightarrow \infty$ (more precisely $1<<\tau<<N$ ), the bracket vanishes: the $\operatorname{PDF} f_{1}$ of the test particle converges toward the quasi-stationary distribution $f_{0}$ of the surrounding bath. This is in complete agreement with the result that $f_{0}$ is stationary for time scales of order $N$.

All the results of this section, except the fact that the Lenard Balescu equation vanishes for one dimensional systems, are classical results. In the next section we explain recent results related to the very interesting and peculiar properties of the Fokker-Planck equation (23). 


\subsection{Autocorrelation function with algebraic decay and anomalous diffusion}

In this subsection, we present recent results [55] which predicted the existence of non exponential relaxation, autocorrelation of the momentum $p$ with algebraic decay at large time, and anomalous diffusion of the spatial or angular variable $x$. They clarify the highly debated disagreement between different numerical simulations reporting either anomalous [64] or normal [65] diffusion, in particular by delimiting the time regime for which such anomalous behavior should occur. We briefly recall that when the moment of order $n$ of the distribution scales like $\tau^{n / 2}$ at large time, such a transport is called normal. However, anomalous transport [94, 95], where moments do not scale as in the diffusive case, were reported in some stochastic models, in continuous time random walks (Levy walks), and for systems with a lack of stationarity of the corresponding stochastic process [96].

These results have been obtained by analyzing theoretically the properties of the Fokker-Planck equation (23). $>$ From the physical point of view, as particles with large momentum $p$ fly very fast in comparison to the typical time scales of the fluctuations of the potential, they experience a very weak diffusion and thus maintain their large momentum during a very long time (one sees from equation (22), using $|\varepsilon(p, 1)|^{2} \underset{p \rightarrow \infty}{\rightarrow}$, that the diffusion coefficient decays as fast as the bath distribution $f_{0}(p)$ for large times). Because of this very weak diffusion for large $p$, the distribution of waiting time for passing from a large value of $p$ to a typical value of $p$, is a thick distribution. This explains the algebraic asymptotic for the correlation function. From a mathematical point of view, these behaviors are linked to the fact that the Fokker-Planck equation (23) has a continuous spectrum down to its ground state (without gap). This leads to a non exponential relaxation of the different quantities and to long-range temporal correlations [55, 97]. These results will generalize to the kinetic theory of any system for which the slow variable (here the momentum) live in an infinite space.

By explicitly deriving an asymptotic expansion of the eigenvalues and eigenfunctions of the Fokker Planck equation, the exponent for the algebraic tail of the autocorrelation function of momenta has been theoretically computed [55, 97]. This mechanism is new in the context of kinetic theory. However, we have discovered later that similar Fokker-Planck equations, with a rapidly vanishing diffusion coefficients obtained by other physical mechanisms, had been studied [98, 99, 100]. A more recent alternative approach to the same phenomena has been proposed [68], together with interesting discussions of kinetic applications.

Let us present the results in the context of the HMF model, for which algebraic large time behaviors for momentum autocorrelations had been first numerically observed in Refs. [69, 66]. In its Quasi Stationary States, the theoretical law for the diffusion of angles $\sigma_{x}^{2}(\tau)$ has been also derived in [55, 97]. The predictions for the diffusion properties are listed in Table 1 .

When the distribution $f_{0}(p)$ is changed within the HMF model, a transition between weak anomalous diffusion (normal diffusion with logarithmic corrections) and strong 
TABLE 1. Asymptotic forms of initial distributions $f_{0}(p)$, and theoretical predictions of correlation functions $C_{p}(\tau)$ and the diffusion $\sigma_{x}^{2}(\tau)$ in the long-time regime. Asymptotic forms of the distribution and the predictions are assumed and predicted in the limits $|p| \rightarrow \infty$ and $\tau \rightarrow \infty$ respectively, where $\tau=t / N$ is a rescaled time. The exponent $\alpha$ is given as $\alpha=(v-3) /(v+2)$. See Ref. [55, 97, for details.

\begin{tabular}{|l|c|c|c|}
\hline Tails & $f_{0}(p)$ & $C_{p}(\tau)$ & $\sigma_{x}^{2}(\tau)$ \\
\hline Power-law & $|p|^{-v}$ & $\tau^{-\alpha}$ & $\tau^{2-\alpha}$ \\
\hline Stretched exponential & $\exp \left(-\beta|p|^{\delta}\right)$ & $\frac{(\ln \tau)^{2 / \delta}}{\tau}$ & $\tau(\ln \tau)^{2 / \delta+1}$ \\
\hline
\end{tabular}

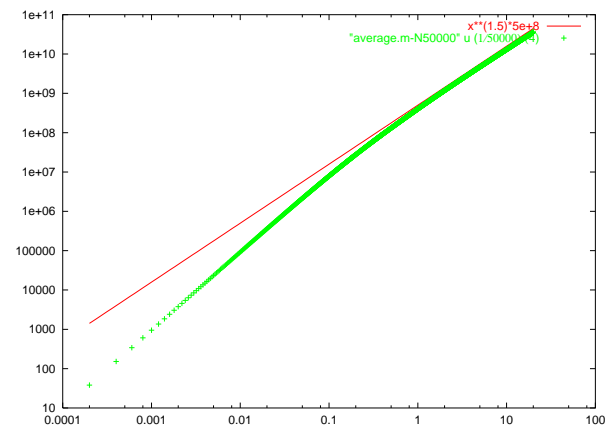

FIGURE 10. Angles diffusion $\left(<(x(t)-x(0))^{2}>\right.$ as a function of time) in the HMF model, for a quasi-stationary state. Points are from a $\mathrm{N}$ body numerical simulation, the straight line is the analytic prediction by the kinetic theory. For large times $\left\langle(x(t)-x(0))^{2}>\underset{t \rightarrow \infty}{\sim} t^{v}\right.$ with $v \neq 1$. Such an unexpected anomalous diffusion is also observed at equilibrium (see [55] for more details)

anomalous diffusion is thus predicted. We have numerically confirmed the theoretical predictions [67]. For initial distributions with power-law or Gaussian tails, correlation function and diffusion are in good agreement with numerical results. Diffusion is indeed anomalous super-diffusion in the case of power-law tails, while normal when Gaussian. In the latter case, the system is at equilibrium, but the diffusion exponent shows a logarithmically slow convergence to unity due to a logarithmic correction of the correlation function. This long transient time to observe normal diffusion, even for Gaussian distribution and at equilibrium, suggests that one should be very careful to decide whether diffusion is anomalous or not.

We note the existence of another interpretation of Quasi Stationary States and anomalous diffusion : the algebraic behaviors for momentum autocorrelations have been fitted using q-exponential functions [69, 66], derived from Tsallis' non extensive statistical mechanics. Our theoretical and numerical results are in disagreement with this interpretation (see [70, 55, 67] for further discussions). By contrast with the use of non extensive 
statistical mechanics, we think that our theory explains the phenomena of long range temporal correlation and of anomalous diffusion from first principles.

\subsection{Challenges in kinetic theories}

Our main message in this section is that a classical kinetic theory approach for these long range interacting systems already explains many intriguing behaviors of these systems. However, in contrast with the equilibrium theory, many questions remain open; we mention here some of them, without any pretention to be exhaustive:

- Can we find a better recipe than Lynden-Bell's theory to predict the outcome of the Vlasov evolution? This seems hopeless in a general setting (see for instance the discussion in [63]).

- Is it possible to explain the 1.7 exponent for the relaxation to equilibrium in the HMF model, and does it have some universality? More generally, is it possible to extract other general features of the dynamics beyond the Vlasov equation, like the anomalous diffusion, or the long relaxation times described above?

- At the mathematical level: is it possible to improve on [80] concerning the lifetime of QSS? Can the convergence theorems to the Vlasov equation be extended to more singular potentials?

- The most important issue concerning kinetic theories is a clear understanding of the limits of validity the different equations. Whereas, for smaller times, kinetic theory are based on solid theoretical arguments, the understanding of larger time behavior of an ensemble of trajectories, initially close to one another, is not yet understood. Numerical computations could be very useful in order to understand that. Very few direct numerical tests of the kinetic theories have been performed up to now. The main reason is probably the difficulty for such tests, because of the long time needed for such test. We think it would be highly relevant to consider such problem, in models as simple as possible.

\section{OUT OF EQUILIBRIUM}

\subsection{Motivations}

We have described the computation of equilibrium states for systems with long range interactions in the first section, and addressed the problem of relaxation to equilibrium in the second one. These two types of problems concern isolated Hamiltonian systems, systems which may be considered so on the relevant time scales, or systems in contact with a thermal bath. In many cases of interest, the system experiences random forces and dissipation. Very often the mechanism for dissipation and random forces are from a different origin, and do not act as a thermal bath. As a consequence, detailed balance is no more valid and the system is subject to fluxes of energy or possibly of other conserved quantities; the average energy of the system is fixed by the balance between forcing and dissipation. The understanding of the properties of the corresponding Non Equilibrium 
Steady States (NESS) is thus of deep importance. We present here first studies of such NESS in the context of systems with long range interactions. The most prominent result is the finding of out of equilibrium phase transitions.

These first studies have been done in the context of two dimensional flows. This is indeed essential in this case, as in many applications of fluid dynamics, one of the most important problem is the prediction of the very high Reynolds' large-scale flows. The highly turbulent nature of such flows, for instance ocean circulation or atmosphere dynamics, renders a probabilistic description desirable, if not necessary. At equilibrium, a statistical mechanics explanation of the self-organization of geophysical flows has been proposed by Robert-Sommeria and Miller (RSM). Out of equilibrium, there are several practical and fundamental problems to understand: How the invariants are selected by the presence of weak forces and dissipation? What are the associated fluctuations? Are all forcings compatible with RSM equilibria?

We will thus study the Navier Stokes equation with weak random stochastic forces and dissipation:

$$
\frac{\partial \omega}{\partial t}+\mathbf{u} \cdot \nabla \omega=v \Delta \omega-\alpha \omega+f_{s}
$$

where $\omega$ is the vorticity, $f_{s}$ is a random force, $\alpha \omega$ is the Rayleigh dissipation and $v$ is the fluid viscosity.

\subsection{Out of equilibrium phase transitions}

In many turbulent geophysical flows, one can see transitions, at random times, between two states with different large scale flows. The most famous example are probably the time reversal of the earth magnetic field. We may cite also experimental studies of such phenomena, for two dimensional magnetic flows [101], rotating tank experiment in relation with weather regimes in meteorology [102], or magnetic field reversal in MHD [103]. In all these examples, this generic phenomenon takes place in systems with a large number of degrees of freedom. The case of simple turbulent flows may be studied in much details theoretically and numerically; we focus here on the case of the two dimensional Navier-Stokes equation with a random force.

Figure [11 shows the relaxation of the 2D Navier-Stokes equation to a statistically stationary state. It illustrates that, depending on the aspect ratio of the domain, two types of large scale flows are possibly observed, either dipoles or unidirectional (zonal) flows. We note that these two topologies are also predicted by the equilibrium statistical theory.

As shown on figure 12, for some values of the control parameter (the aspect ratio of the domain), we observe the coexistence of these two flow topologies: the system switches back and forth, at random times, between dipole and unidirectional flows. This phenomenology is similar to what happens when noise is added to a bistable system. A crucial difference here, is that the deterministic dynamics does not have two different attracting states (there is no double well potential in this case).

These few figures show that NESS, for systems with long range interactions, may exhibit very interesting phenomena. We hope that this will open a large number of new 

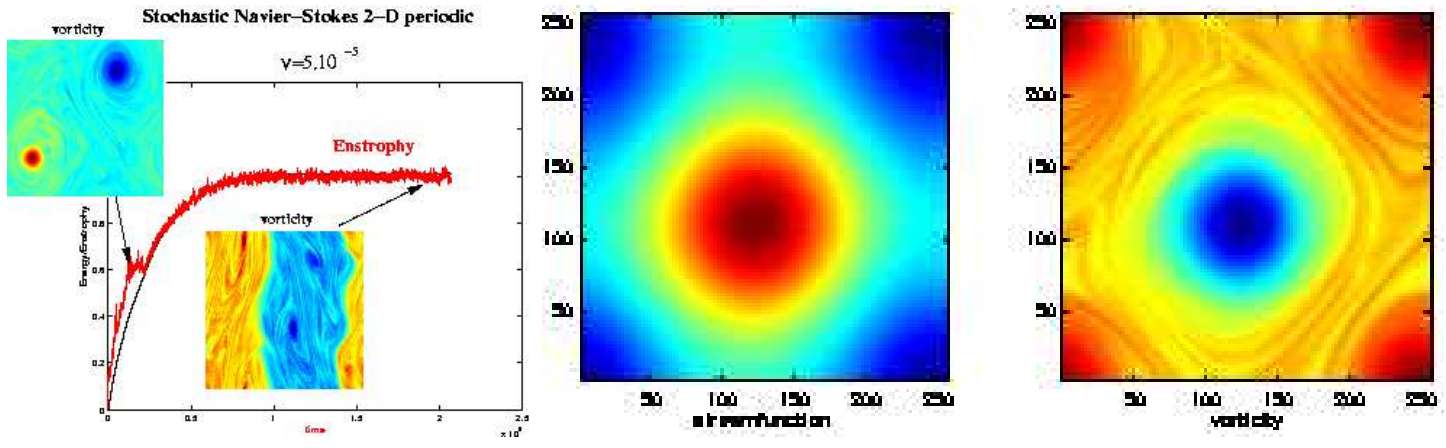

FIGURE 11. Right panel: relaxation towards a statistically stationary state of the two dimensional Navier Stokes equation. After a transient state with a dipole vorticity field, the flow switches to a zonal (unidirectional) organization of the vorticity field. Left panel: for a different value of the control parameter (here the aspect ratio of the domain), we observe a dipole organization in a statistically stationary situation.
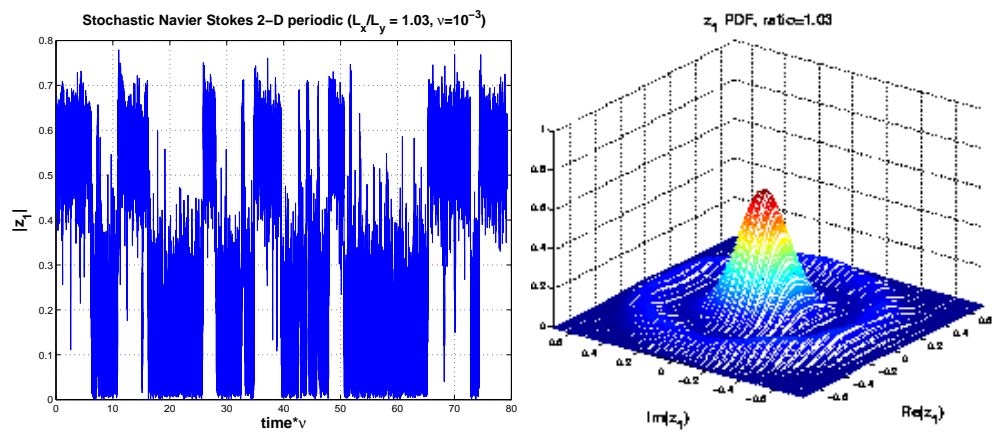

FIGURE 12. Right panel: $\left|z_{1}\right|$, the modulus of the first Fourier mode of the vorticity field in the direction parallel to the unidirectional flow (for unidirectional flows, $\left|z_{1}\right|$ is close to 0 , while for dipoles it oscillates around 0.5 . The flow thus switches randomly from dipole to unidirectional flows. Left panel: PDF of the complex variable $z_{1}$.

fundamental works on the subject. Moreover, in a forthcoming paper, we will discuss the application of these results to geophysical flows.

\section{CONCLUSION}

We have briefly reviewed in this contribution old and new results on the old, but active, subject of systems with long range interactions. We clearly acknowledge that this review is far from exhaustive and represents our personal interests.

In conclusion, it seems to us that equilibrium statistical mechanics of these non additive systems is very well understood: a careful application of standard tools allows one to deal with the unusual non additivity condition, see the section devoted to equilibrium. The situation is somewhat similar as far as relaxation to equilibrium is concerned: in this case also, classical tools, namely those of kinetic theory, have proved sufficient to explain some unexpected phenomena. Thus, we fell that there is at present no obvious 
need for an alternative theory describing the relaxation of these systems with long range interacting.

Let us note that despite the successes of these well established theories, standard statistical mechanics at equilibrium, classical kinetic theory concerning the relaxation to equilibrium, there remains open questions and challenges, and room for new discoveries, especially concerning the relaxation; we have tried to outline a few of them along the way. The most important are probably, on one hand the quest for ensemble inequivalence, negative specific heat and phase transitions in natural phenomena or laboratory experiment, and on the other hand the understanding of the limits of kinetic theories. However, we feel that the most relevant questions, both theoretically and practically, concern forced and dissipative systems, out of equilibrium. The last section presents very recent preliminary steps towards an undertanding of these situations, for which the theory is far less developed.

\section{ACKNOWLEDGMENTS}

We warmly thank P.H. Chavanis, G. De Ninno, D. Fanelli, K. Jain, D. Mukamel, J. Sommeria, T. Tatekawa and Y. Yamaguchi for their participation to some of the works described in this proceeding. We thank especially T. Dauxois and S. Ruffo for their role in rising our interests for systems with long range interactions, and for the active collaborations we had during the last years, in Firenze and in Lyon.

Our thought also go to Dieter Gross, the partner of so many discussions, and whom we remember fondly and vividly.

This work was supported by the ANR program STATFLOW (ANR-06-JCJC-0037$01)$.

\section{REFERENCES}

1. $\quad$ D. Lynden-Bell, and R. Wood, Mon. Not. R. Astron. Soc. 138, 495 (1968).

2. $\quad$ P. Klinko, and B. N. Miller, Phys. Rev. E 65, 056127 (2002).

3. V. P. Youngkins, and B. N. Miller, Phys. Rev. E 62, 4583-4596 (2000).

4. B. Stahl, M. K.-H. Kiessling, and K. Schindler, Planet. Space Sci. 43, 271-282 (1995).

5. $\quad$ B. N. Miller, and P. Youngkins, Phys. Rev. Lett. 81, 4794-4797 (1998).

6. D. H. E. Gross, and E. V. Votyakov, Eur. Phys. J. B 15, 115-126 (2000).

7. P. H. Chavanis, and M. Rieutord, Astronomy and Astrophysics 412, 1-17 (2003).

8. D. Heggie, and P. Hut, The Gravitational Million-Body Problem, Cambridge University Press, 2003.

9. I. Cohen, E.G. D. Ispolatov, "Phase Transitions in Systems with $1 / r^{\alpha}$ Attractive Interactions," in Dynamics and Thermodynamics of Systems With Long Range Interactions, edited by T. Dauxois, S. Ruffo, E. Arimondo, and M. Wilkens, 2002.

10. N. De Vega, H.J. Sanchez, Nucl. Phys. B 625, 409-459 (2002).

11. W. Hertel, P. Thirring, Annals Phys. 63, 520-533 (1971).

12. E. Caglioti, P. L. Lions, C. Marchioro, and M. Pulvirenti, Commun. Math. Phys. 174, 229-260 (1995).

13. M. Kiessling, and J. Lebowitz, Lett. Math. Phys. 42, 43-58 (1997).

14. J. Sommeria, and R. Robert, J. Fluid Mech. 229, 291-310 (1991).

15. J. Miller, Phys. Rev. Lett. 65, 2137-2140 (1990). 
16. P. Chavanis, "Statistical Mechanis of Two-Dimensional Vortices and Stellar Systems," in Dynamics and Thermodynamics of Systems With Long Range Interactions, edited by T. Dauxois, S. Ruffo, E. Arimondo, and M. Wilkens, 2002.

17. F. Bouchet, and J. Sommeria, J. Fluid. Mech. 464, 465-207 (2002).

18. Y. Elkens, and D. Escande, Microscopic Dynamics of Plasmas and Chaos, IoP Publishing, Bristol, 2002.

19. M. K.-H. Kiessling, and T. Neukirch, Proc. Nat. Acad. Sci. 100, 1510-1514 (2003).

20. M.-C. Firpo, and Y. Elskens, Phys. Rev. Lett. 84, 3318-3321 (2000).

21. J. Barré, T. Dauxois, G. de Ninno, D. Fanelli, and S. Ruffo, Phys. Rev. E 69, 045501-+ (2004).

22. J. Barré, D. Mukamel, and S. Ruffo, Phys. Rev. Lett. 87, 030601 (2001).

23. T. Dauxois, S. Ruffo, E. Arimondo, and M. Wilkens, editors, Dynamics and Thermodynamics of Systems With Long Range Interactions, 2002.

24. M. Antoni, and S. Ruffo, Phys. Rev. E 52, 2361-2374 (1995).

25. M. Antoni, S. Ruffo, and A. Torcini, Phys. Rev. E 66, 025103-+ (2002).

26. F. Bouchet, and J. Barré, J. Stat. Phys. 118 5/6, 1073-1105 (2005).

27. P. Hertel, and W. Thirring, Commun. Math. Phys. 24, 22-36 (1971).

28. R. A. Smith, and T. M. O’Neil, Phys. Fluids B 2, 2961-2975 (1990).

29. R. S. Ellis, K. Haven, and B. Turkington, Nonlinearity 15, 239-255 (2002).

30. T. Tatekawa, F. Bouchet, T. Dauxois, and S. Ruffo, Phys. Rev. E 71, 056111-+ (2005).

31. O. Iguchi, Y. Sota, T. Tatekawa, A. Nakamichi, and M. Morikawa, Phys. Rev. E 71, 016102 (2005).

32. Y. Sota, O. Iguchi, M. Morikawa, T. Tatekawa, and K.-I. Maeda, Phys. Rev. E 64, 056133 (2001).

33. F. Tamarit, and C. Anteneodo, Phys. Rev. Lett. 84, 208-211 (2000).

34. M. Grousson, G. Tarjus, and P. Viot, Phys. Rev. E 62, 7781-7792 (2000).

35. E. Kac, G. Uhlenbeck, and H. P. C., Jour. Math. Phys. 4, 216-228 (1963).

36. Y. Messer, and H. Spohn, J. Stat. Phys. 29 (1982).

37. R. S. Ellis, K. Haven, and B. Turkington, J. Stat. Phys. 101, 999 (2000).

38. R. S. Ellis, H. Touchette, and B. Turkington, Physica A 335, 518-538 (2004).

39. R. Boucher, C. Ellis, and B. Turkington, Annals Prob. 27, 297-324 (1999).

40. H. Touchette, R. S. Ellis, and B. Turkington, Physica A 340, 138-146 (2004).

41. J. Barré, F. Bouchet, T. Dauxois, and S. Ruffo, J. Stat. Phys. 119 1/2 (2005).

42. F. Bouchet, ArXiv e-prints (2007), 0710.5094 .

43. P.-H. Chavanis, Phys. Rev. E 68, 036108 (2003).

44. P.-H. Chavanis, Physica D 200, 257-272 (2005).

45. V. I. Arnold, Izv. Vyssh. Uchebbn. Zaved. Matematika; Engl. transl.: Am. Math. Soc. Trans. 79, 267-269 (1966).

46. D. D. Holm, J. E. Marsden, T. Ratiu, and A. Weinstein, Phys. Rep. 123, 1-2 (1985).

47. J. Michel, and R. Robert, J. Stat. Phys. 77, 645-666 (1994).

48. F. Bouchet, Mécanique statistique des écoulements géophysiques, PHD, Université Joseph FourierGrenoble, 2001.

49. F. Bouchet, and T. Dumont, cond-mat/0305206 (2003).

50. A. Venaille, and F. Bouchet, ArXiv e-prints 710 (2007), 0710.5606.

51. P. H. Chavanis, and J. Sommeria, J. Fluid Mech. 314, 267-297 (1996).

52. H. Touchette, and C. Beck, J. Stat. Phys. 125, 455-471 (2006).

53. L. Spitzer, Dynamical evolution of Globular Clusters, Princeton University Press, 1991.

54. D. Nicholson, Introduction to plasma theory, Wiley, New-York, 1983.

55. F. Bouchet, and T. Dauxois, Phys. Rev. E 72, 045103-+ (2005).

56. D. H. E. Dubin, and T. M. O’Neil, Phys. Rev. Lett. 60, 1286-1289 (1988).

57. D. H. E. Dubin, Phys. Plasmas 10, 1338-1350 (2003).

58. V. Latora, A. Rapisarda, and S. Ruffo, Phys. Rev. Lett. 80, 692-695 (1998).

59. Y. Yamaguchi, J. Barré, F. Bouchet, T. Dauxois, and S. Ruffo, Physica A 337 (1-2), $36-66$ (2004).

60. D. H. E. Dubin, and T. M. O'Neil, Rev. Mod. Phys. 71, 87 (1999).

61. J. Barré, T. Dauxois, G. de Ninno, D. Fanelli, and S. Ruffo, Phys. Rev. E 69, 045501 (2004).

62. A. Antoniazzi, Y. Elskens, D. Fanelli, and S. Ruffo, Eur. Phys. J. B 50, 603-611 (2006).

63. P.-H. Chavanis, Physica A 365, 102-107 (2006).

64. V. Latora, A. Rapisarda, and S. Ruffo, Phys. Rev. Lett. 83, 2104-2107 (1999).

65. Y. Y. Yamaguchi, Phys. Rev. E 68, 066210 (2003). 
66. A. Pluchino, V. Latora, and A. Rapisarda, Physica D Nonlinear Phenomena 193, 315-328 (2004).

67. Y. Y. Yamaguchi, F. Bouchet, and T. Dauxois, J. Stat. Mech. 1,20-+(2007), arXiv : cond-mat/ 0701366.

68. P.-H. Chavanis, and M. Lemou, Phys. Rev. E 72, 061106 (2005).

69. V. Latora, A. Rapisarda, and C. Tsallis, Phys. Rev. E 64, 056134 (2001).

70. F. Bouchet, T. Dauxois, and S. Ruffo, Europhysics News 37, 9-10 (2006).

71. M.-C. Firpo, Phys. Rev. E 57, 6599-6603 (1998).

72. F. Borgonovi, G. L. Celardo, M. Maianti, and E. Pedersoli, J. Stat. Phys. 116 (2004).

73. G. L. Celardo, J. Barré, F. Borgonovi, and S. Ruffo, Phys. Rev. E 73, 011108 (2006).

74. F. Bouchet, T. Dauxois, D. Mukamel, and S. Ruffo, ArXiv e-prints (2007), 0711.0268 .

75. D. del-Castillo-Negrete, Physica A 280, 10-21 (2000).

76. W. Braun, and K. Hepp, Commun. Math. Phys. 56, 101-113 (1977).

77. M. Hauray, and P.-E. Jabin, Arch. Rat. Mech. Anal. 183, 489-524 (2007).

78. K. Jain, F. Bouchet, and D. Mukamel, J. Stat. Mech. (2007).

79. D. Mukamel, S. Ruffo, and N. Schreiber, Phys. Rev. Lett. 95, 240604 (2005).

80. E. Caglioti, and F. Rousset, J. Stat. Phys. 129, 241-263 (2007).

81. J. Goodman, T. Y. Hou, and J. Lowengrub, Comm. Pure Appl. Math. 43, 415-430 (1990).

82. M. C. Firpo, and Y. Elskens, J. Stat. Phys. 93, 193-209 (1998).

83. A. Taruya, and M.-A. Sakagami, Phys. Rev. Lett. 90, 181101 (2003).

84. M.-C. Firpo, F. Doveil, Y. Elskens, P. Bertrand, M. Poleni, and D. Guyomarc'h, Phys. Rev. E 64, 026407-+ (2001).

85. D. D. Holm, J. E. Marsden, T. Ratiu, and A. Weinstein, Phys. Rep. 123, 1-2 (1985).

86. A. Campa, A. Giansanti, D. Mukamel, and S. Ruffo, Physica A 365, 120-127 (2006).

87. F. Baldovin, and E. Orlandini, Phys. Rev. Lett. 96, 240602 (2006).

88. P. H. Chavanis, J. Sommeria, and R. Robert, Astro. Phys. Jour. 471, 385 (1996).

89. R. Robert, J. Stat. Phys. 65, 531-553 (1991).

90. I. Arad, and P. H. Johansson, Mon. Not. R. Astron. Soc. 362, 252-262 (2005).

91. E. M. Lifshitz, and L. P. Pitaevskii, Physical kinetics, Course of theoretical physics, Oxford: Pergamon Press, 1981, 1981.

92. J. Binney, and S. Tremaine, Galactic dynamics, Princeton, NJ, Princeton University Press, 1987, 747 p., 1987.

93. H. Spohn, Large Scale Dynamics of Interacting Particles, Springer, New-York, 2002.

94. J.-P. Bouchaud, and A. Georges, Phys. Rep. 195, 127-293 (1990).

95. P. Castiglione, A. Mazzino, P. Muratore-Ginanneschi, and A. Vulpiani, Physica D 134, 75-93 (1999).

96. F. Bouchet, F. Cecconi, and A. Vulpiani, Phys. Rev. Lett. 92, 040601 (2004).

97. F. Bouchet, and T. Dauxois, Journal of Physics: Conference Series 7, 34-47 (2005).

98. J. Farago, Europhys. Lett. 52, 379-385 (2000).

99. F. Lillo, S. Micciche, and R. N. Mantegna, ArXiv (2002), cond-mat/02034 42.

100. E. Lutz, Phys. Rev. Lett. 93, 190602 (2004).

101. J. Sommeria, J. Fluid. Mech. 170, 139-168 (1986).

102. Y. Tian, E. R. Weeks, K. Ide, J. S. Urbach, C. N. Baroud, M. Ghil, and H. L. Swinney, J. Fluid Mech. 438, 129-157 (2001).

103. M. Berhanu, R. Monchaux, S. Fauve, N. Mordant, F. Petrelis, A. Chiffaudel, F. Daviaud, B. Dubrulle, L. Marie, F. Ravelet, M. Bourgoin, P. Odier, J. . Pinton, and R. Volk, Eur. Phys. Lett. (2007). 